OAK RIDGE

NATIONAL LABORATORY

ORNL/TM-2010/60

MANAGED BY UT-BATTELLE

FOR THE DEPARTMENT OF ENERGY

\title{
Getting Started with VIBE as a DICE Plug-in Module
}

\section{August 2010}

Prepared by

B. T. Rearden

R. A. Lefebvre

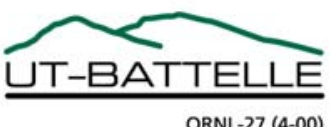

ORNL-27 (4-00) 


\title{
DOCUMENT AVAILABILITY
}

Reports produced after January 1, 1996, are generally available free via the U.S. Department of Energy (DOE) Information Bridge.

Web site http://www.osti.gov/bridge

Reports produced before January 1, 1996, may be purchased by members of the public from the following source.

\author{
National Technical Information Service \\ 5285 Port Royal Road \\ Springfield, VA 22161 \\ Telephone 703-605-6000 (1-800-553-6847) \\ TDD 703-487-4639 \\ Fax 703-605-6900 \\ E-mail info@ntis.gov \\ Web site http://www.ntis.gov/support/ordernowabout.htm
}

Reports are available to DOE employees, DOE contractors, Energy Technology Data Exchange (ETDE) representatives, and International Nuclear Information System (INIS) representatives from the following source.

Office of Scientific and Technical Information

P.O. Box 62

Oak Ridge, TN 37831

Telephone 865-576-8401

Fax 865-576-5728

E-mail reports@osti.gov

Web site http://www.osti.gov/contact.html

\begin{abstract}
This report was prepared as an account of work sponsored by an agency of the United States Government. Neither the United States Government nor any agency thereof, nor any of their employees, makes any warranty, express or implied, or assumes any legal liability or responsibility for the accuracy, completeness, or usefulness of any information, apparatus, product, or process disclosed, or represents that its use would not infringe privately owned rights. Reference herein to any specific commercial product, process, or service by trade name, trademark, manufacturer, or otherwise, does not necessarily constitute or imply its endorsement, recommendation, or favoring by the United States Government or any agency thereof. The views and opinions of authors expressed herein do not necessarily state or reflect those of the United States Government or any agency thereof.
\end{abstract}


ORNL/TM-2010/60

Nuclear Science and Technology Division

\title{
GETTING STARTED WITH VIBE AS A DICE PLUG-IN MODULE
}

B.T. Rearden

R.A. Lefebvre

Date Published: August 2010

\author{
Prepared by \\ OAK RIDGE NATIONAL LABORATORY \\ P.O. Box 2008 \\ Oak Ridge, Tennessee 37831-6285 \\ managed by \\ UT-BATTELLE, LLC \\ for the \\ U.S. DEPARTMENT OF ENERGY \\ under contract DE-AC05-00OR22725
}





\section{CONTENTS}

PAGE

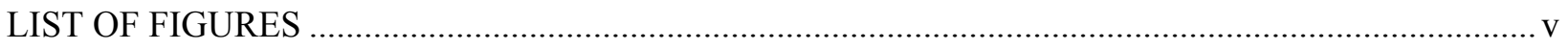

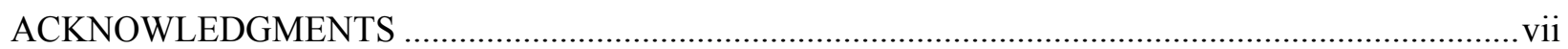

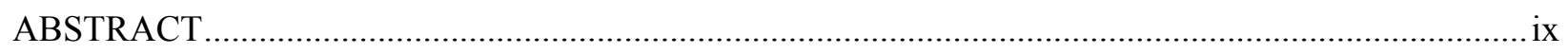

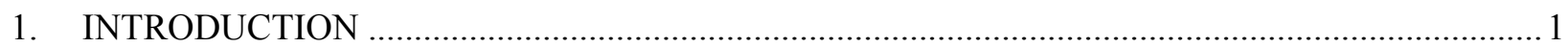

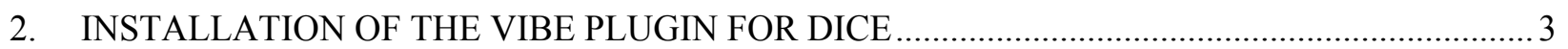

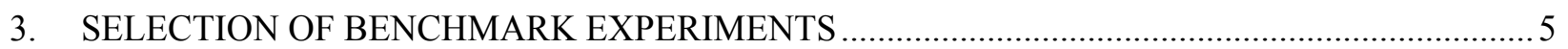

3.1 SENSITIVITY DATA DISTRIBUTED WITH ICSBEP HANDBOOK .............................5

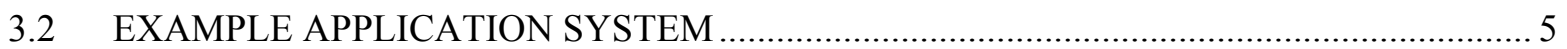

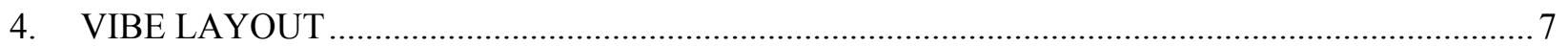

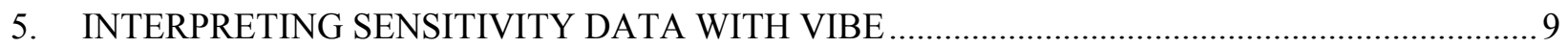

5.1 QUANTIFICATION OF IMPORTANT PROCESSES IN THE APPLICATION ............... 10

5.2 INITIAL SCREENING OF AVAILABLE EXPERIMENTS WITH VIBE.......................21

6. EXPORTING GROUP-COLLAPSED SENSITIVITY DATA............................................41

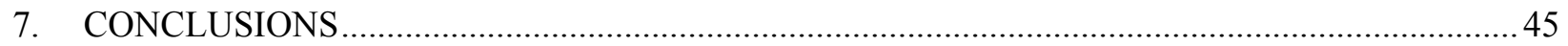

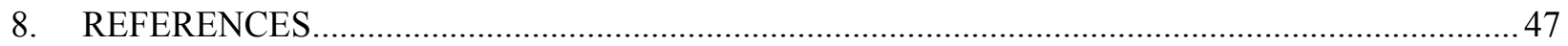





\section{LIST OF FIGURES}

Page

Fig. 1. ICSBEP data installed in C: Handbook Directory ....................................................... 3

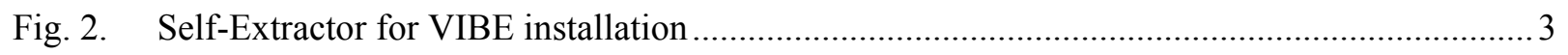

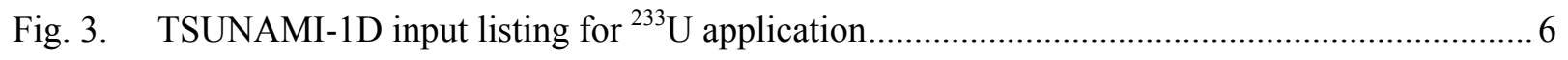

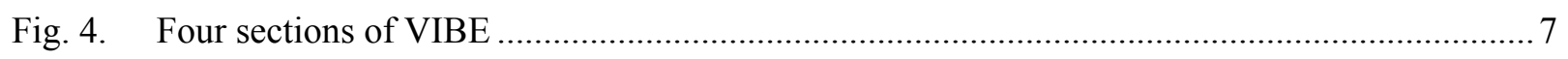

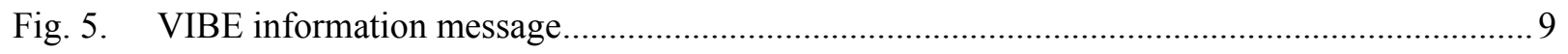

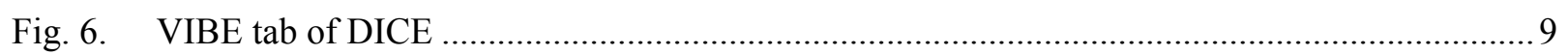

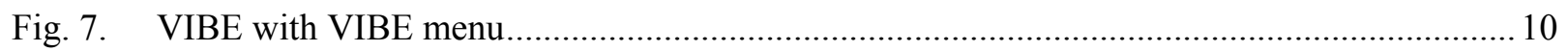

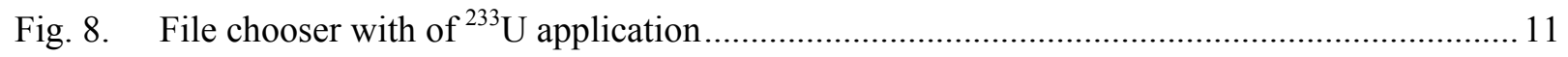

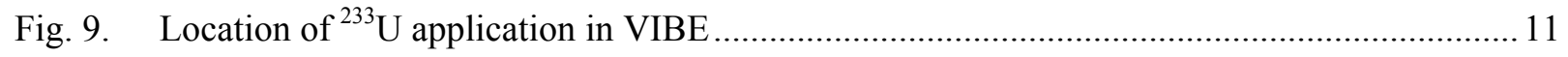

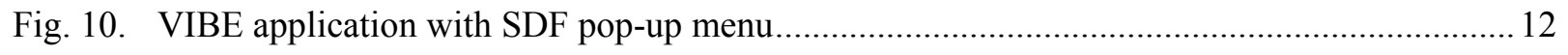

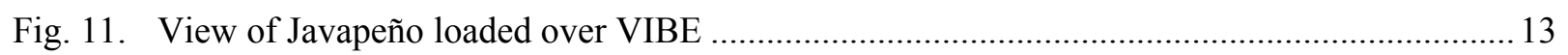

Fig. 12. Plotting ${ }^{233} \mathrm{U}$ fission and $\mathrm{n}$,gamma sensitivities for the ${ }^{233} \mathrm{U}$ application .............................. 14

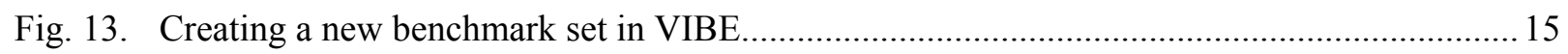

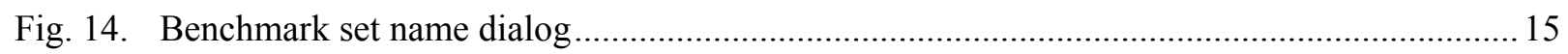

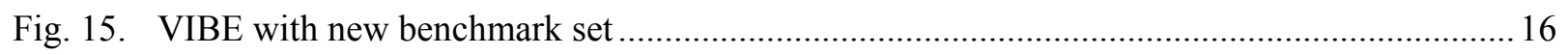

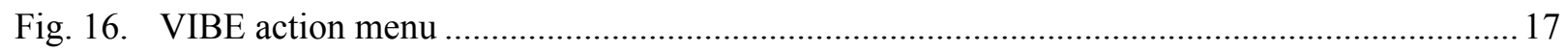

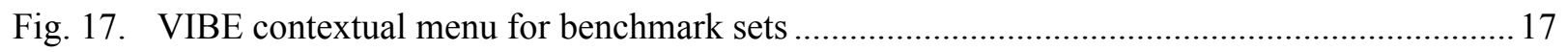

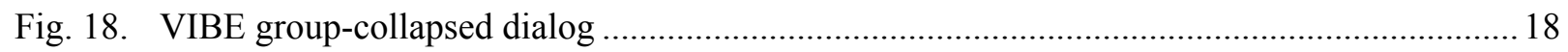

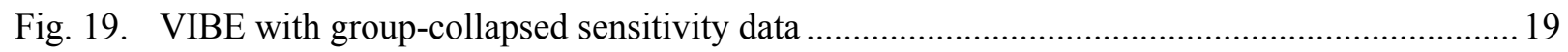

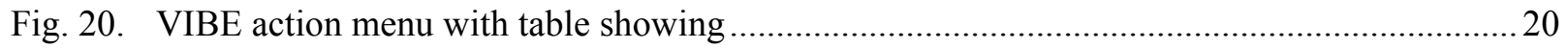

Fig. 21. VIBE sort dialog for sorting by thermal sensitivities by absolute in descending order............20

Fig. 22. VIBE with the absolute values of the thermal sensitivity data sorted in descending order ......21

Fig. 23. VIBE Sensitivity File Panel with ICSBEP filter applied ........................................................ 22

Fig. 24. Creation of new benchmark set for data distributed with ICSBEP Handbook .........................22

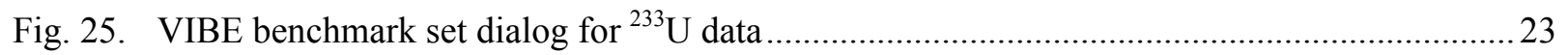

Fig. 26. VIBE with group-collapsed sensitivity data for benchmarks from the ICSBEP Handbook .... 23

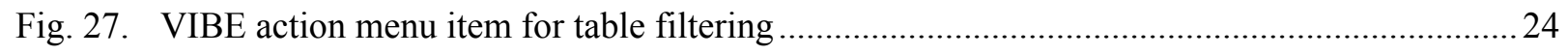

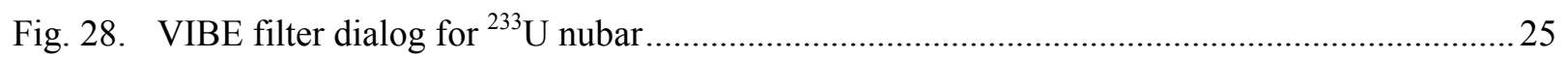

Fig. 29. VIBE with ${ }^{233} \mathrm{U}$ filter applied to ICSBEP Handbook data .................................................. 26

Fig. 30. VIBE with ${ }^{233} \mathrm{U}$ nubar filter and descending sorting for thermal sensitivities applied to ICSBEP Handbook data 26 


\section{LIST OF FIGURES (continued)}

Page

Fig. 31. Values near 0.69 from VIBE with ${ }^{233} \mathrm{U}$ filter and descending sorting for thermal

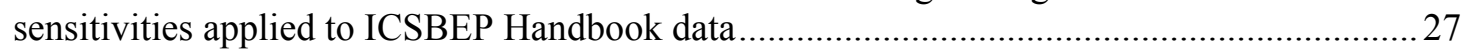

Fig. 32. VIBE Filter dialog for thermal ${ }^{233} U$ nubar sensitivities with values $\geq 0.67$ and $\leq 0.71 \ldots \ldots \ldots .27$

Fig. 33. VIBE with ${ }^{233} \mathrm{U}$ nubar thermal sensitivities near 0.69 filter applied to ${ }^{233} \mathrm{U}$ benchmarks........ 28

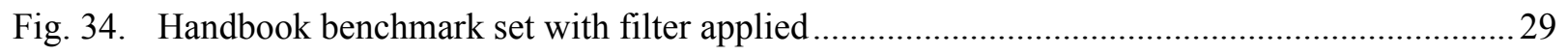

Fig. 35. Javapeño reaction list with data for ${ }^{233} U$ application and U233-SOL-THERM-016-003 ......... 29

Fig. 36. Javapeño reaction list for plotting ${ }^{233} U$ nubar sensitivity data for ${ }^{233} U$ application

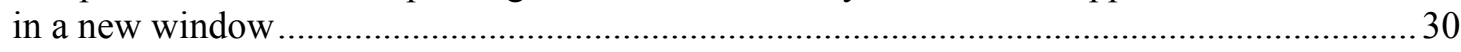

Fig. 37. Javapeño reaction list for plotting ${ }^{233} \mathrm{U}$ nubar sensitivity data for

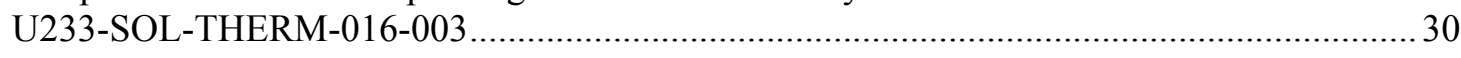

Fig. 38. Javapeño plot of ${ }^{233} \mathrm{U}$ nubar sensitivities for ${ }^{233} \mathrm{U}$ application and

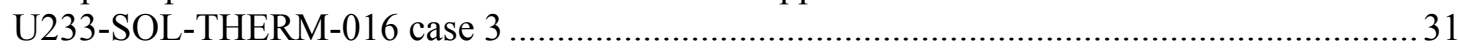

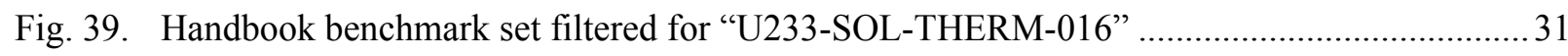

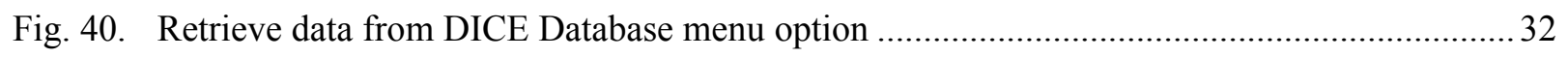

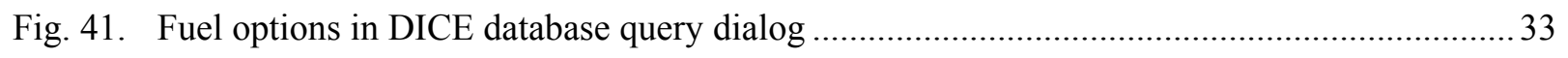

Fig. 42. Benchmark $k_{\text {eff }}$ and uncertainty options in DICE database query dialog ............................... 33

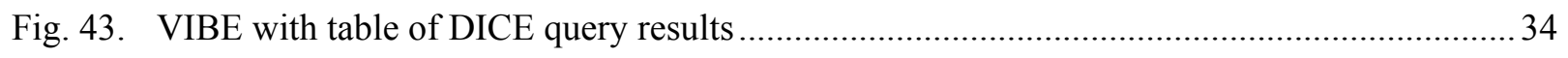

Fig. 44. Benchmarks window with U233-SOL-THERM filter applied ............................................. 35

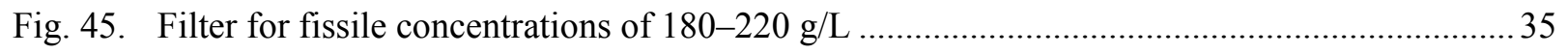

Fig. 46. VIBE DICE query results for all U233-SOL-THERM experiments filtered from

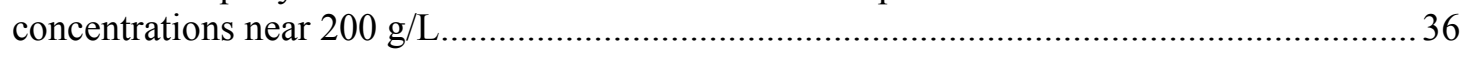

Fig. 47. VIBE DICE query results for U233-SOL-THERM-002 benchmarks .................................... 37

Fig. 48. Sensitivity profiles for ${ }^{233} \mathrm{U}$ nubar from ${ }^{233} \mathrm{U}$ application and

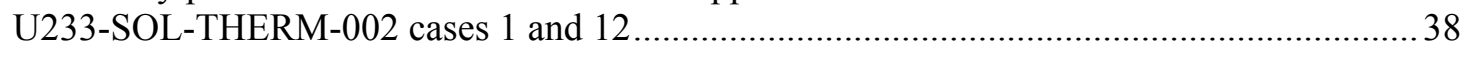

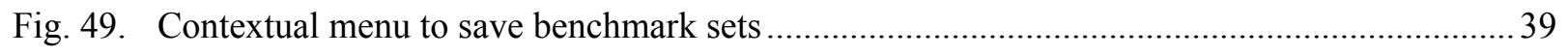

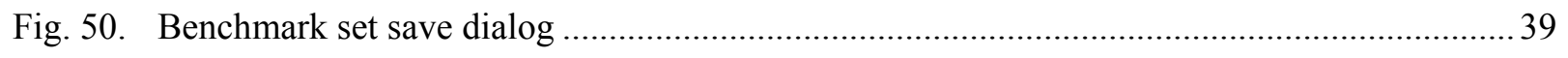

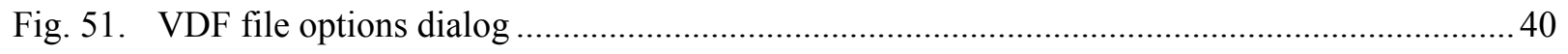

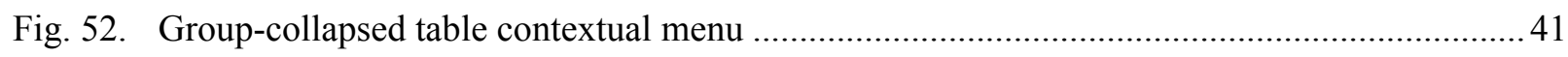

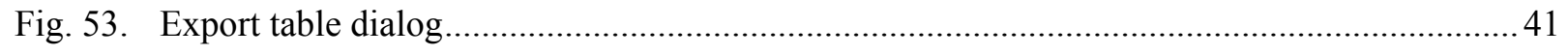

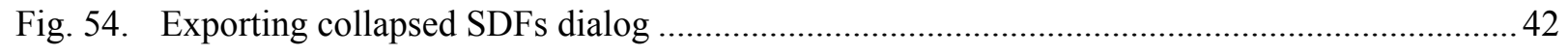

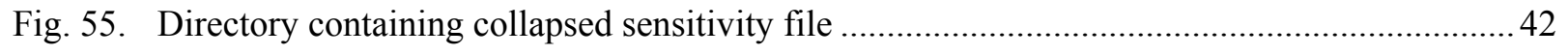

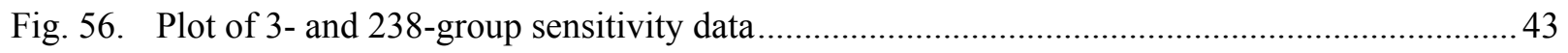




\section{ACKNOWLEDGMENTS}

The authors wish to acknowledge the technical assistance of the DICE developers, Manuel Bossant and Nicolas Soppera, in developing the DICE plug-in interface and assisting with the VIBE database reading routines. The authors also acknowledge Adam Thompson, the original author of VIBE, who worked at Oak Ridge National Laboratory through the Nuclear Engineering Science Laboratory Synthesis Program while completing his graduate studies in computer science at the University of Tennessee. The assistance of the International Criticality Safety Benchmark Evaluation Project Program Manager Blair Briggs of Idaho National Laboratory and Nichole Ellis of Ellis Nuclear Engineering, LLC in testing the integration of VIBE in DICE is gratefully acknowledged. The U.S. Department of Energy Nuclear Criticality Safety Program supported this work. 



\begin{abstract}
The use of the SCALE (Standardized Computer Analysis for Licensing Evaluation) tool VIBE (Validation, Interpretation and Bias Estimation) as a plug-in module to DICE (Database for the International Criticality Safety Benchmark Evaluation Project) to access and interpret sensitivity data is described. In this initial release of VIBE within DICE, VIBE can identify sensitivity data and group collapse the data into a table, where the values can be sorted and filtered to identify experiments that are the most similar to a targeted application system in terms of sensitivity data. VIBE can then retrieve information about the selected experiments from the DICE database to provide the physical characteristics of the benchmarks.
\end{abstract}





\section{INTRODUCTION}

VIBE (Validation, Interpretation and Bias Estimation) ${ }^{1}$ is a new graphical tool developed for

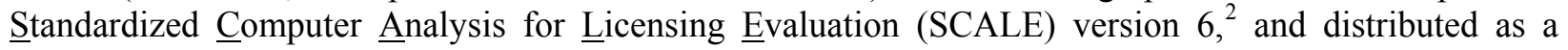
plug-in module for DICE (Database for the International Criticality Safety Benchmark Evaluation Project). ${ }^{3}$ VIBE assists with the interpretation of sensitivity data distributed with DICE, including the 30-group ABBN-93 sensitivity data generated by Institute for Physics and Power Engineering (IPPE) and the 238-group ENDF/B-VII.0 sensitivity data generated with the Tools for Sensitivity and Uncertainty Analysis Methodology Implementation (TSUNAMI) tools of SCALE 6 by Oak Ridge National Laboratory (ORNL). This guide is designed to demonstrate the most common functions of VIBE in a step-by-step guide with numerous illustrations to clarify the mechanics of applying the tools. The example problems have been selected to represent real systems yet be simple enough that the interested reader can quickly reproduce the problems while reading the guide. The information presented here is by no means an exhaustive review of all possible applications of VIBE, and most applications will be more complex than the example presented herein. Furthermore, the examples presented in the document reflect the use of data from the 2009 edition of DICE. Some variation in the results may be obtained when using the 2010 edition of DICE. 



\section{INSTALLATION OF THE VIBE PLUGIN FOR DICE}

For the 2010 edition of the International Criticality Safety Benchmark Evaluation Project (ICSBEP) DVD, VIBE is an integral componet of DICE an further installion is not necessary. For the 2009 edition of ICSBEP, VIBE is an optional plugin for DICE which is available for download from the SCALE website at http://www.ornl.gov/sci/scale/vibe/download.html. The installation of VIBE requires that DICE be available on a writeable hard disk, not on the DVD. In this example, the contents of the ICSBEP DVD have been copied into a local directory, C: Handbook, as shown in Fig. 1. For Windows installation, download the file VIBE_plugin_for_DICE.exe, as self-extracting ZIP archive, and double-click on the file. If DICE is installed in another location, direct the installer to directory that encloses the DICE directory from the Handbook. Once the directory that contains the DICE directory is selected, press the "Unzip" button on the self-extractor application to install VIBE as a DICE plugin, as shown in Fig. 2.

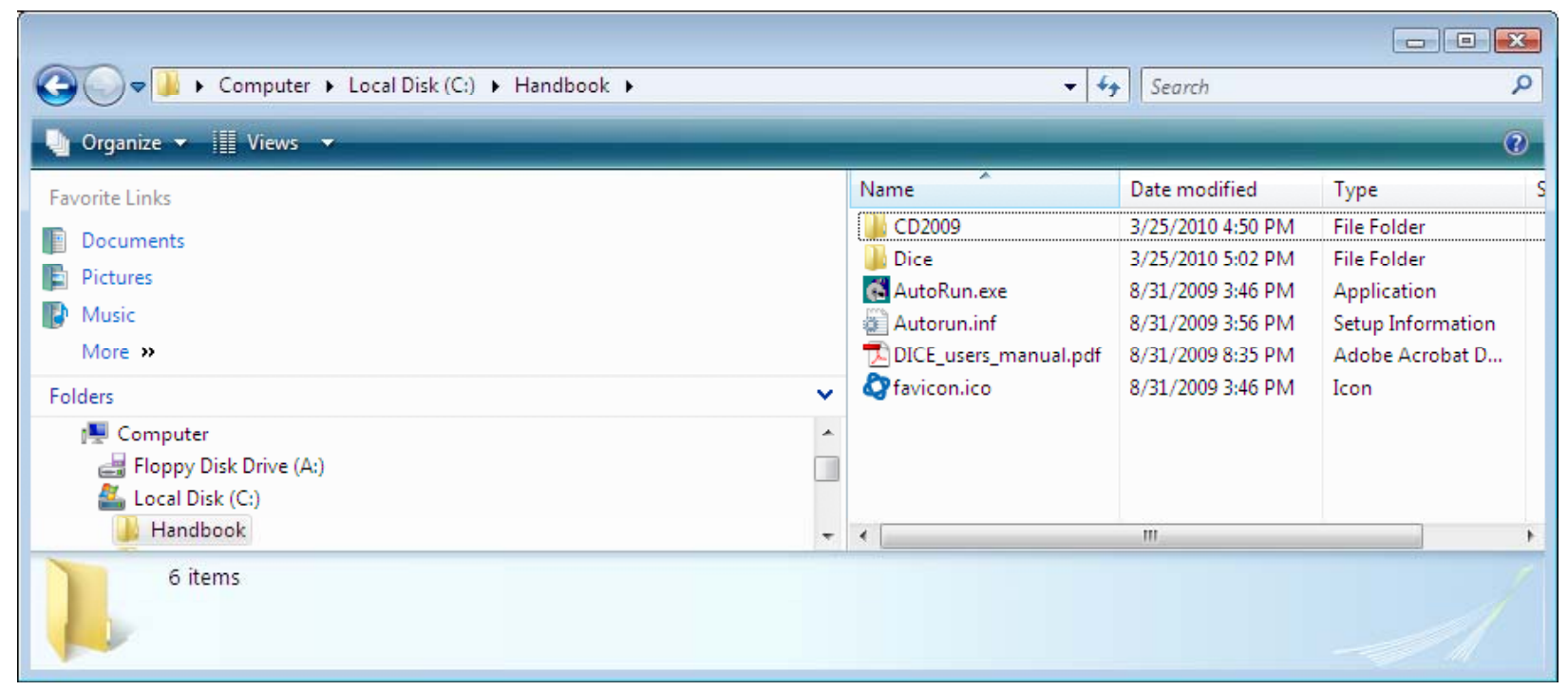

Fig. 1. ICSBEP data installed in C: $\mid$ Handbook Directory.

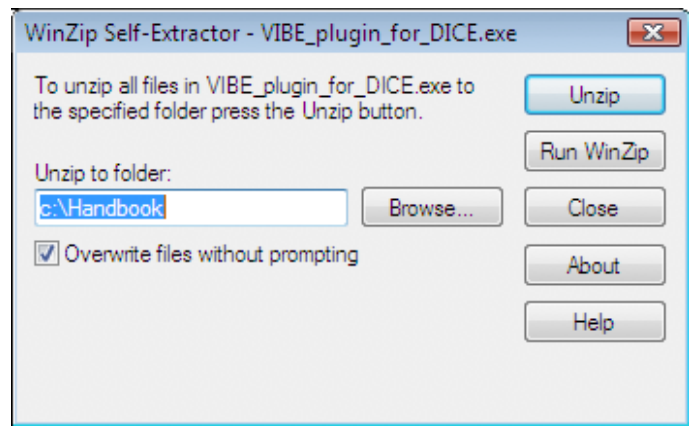

Fig. 2. Self-Extractor for VIBE installation. 



\section{SELECTION OF BENCHMARK EXPERIMENTS}

The accurate generation and interpretation of sensitivity and uncertainty data for individual systems can help the user gain insight into which processes are important and which processes may be the primary contributors to computational bias. The VIBE tool, included as a plug-in component of DICE, allows the use of sensitivity information as a means of comparing systems to identify benchmark experiments that are similar to the targeted application. The premise behind this concept is that computational biases are primarily due to errors in cross-section data. If a system has a high sensitivity in a given energy range to a cross section with large errors, the system is likely to exhibit a computational bias. If a benchmark experiment is identified that has a similar sensitivity to that of the application, the computational bias can be quantified with the benchmark, and the bias can be projected to the application system. This manual describes how to use VIBE to assist in identifying important processes in the application system and finding benchmark experiments that can be useful in their validation.

\subsection{SENSITIVITY DATA DISTRIBUTED WITH ICSBEP HANDBOOK}

Because of the wide use of benchmark experiments from the IHECSBE for criticality safety validations, an effort is under way to distribute sensitivity data for many benchmarks through the Handbook itself. For the 2009 edition, sensitivity data for several hundred benchmark experiments were generated with SCALE 6 using ENDF/B-VII.0 cross-section data. Many of the inputs were generated as part of a study published as Application of the SCALE TSUNAMI Tools for the Validation of Criticality Safety Calculations Involving ${ }^{233}$ U, ORNL/TM-2008/196. ${ }^{4}$ These input files were generated by expert users of SCALE, but were not subjected to a rigorous peer review process.

Additional input files have been generated as part of an effort jointly supported by the U.S. Department of Energy Nuclear Criticality Safety Program and the U.S. Nuclear Regulatory Commission. For this project, a new SCALE procedure was created to guide the development of Models and Derived Data (MADD). The MADD procedure requires input models to match the description provided in Section 3 of an IHECSBE benchmark evaluation to the extent possible using multi-group cross sections and three-dimensional (3D) modeling with KENO V.a or KENO-VI. The models are generated by an expert originator and independently checked by an expert reviewer. Additionally, the sensitivity data generated through TSUNAMI calculations are rigorously checked with direct perturbation calculations to ensure that the data are accurate. Models are often refined after direct perturbation results reveal inadequate resonance self-shielding models or inadequate spatial resolution of the flux solution through the use of mesh flux accumulators. The input models and sensitivity results are only accepted into MADD archive after they have passed this rigorous assessment of quality by the originator and reviewer.

The TSUNAMI sensitivity data are located in the directories Dice/data/ornI/TSUNAMI-1D and Dice/data/ornl/TSUNAMI-3D. Input files for systems that have passed through the MADD process are also distributed in Dice/data/ornl/inputs.

The IPPE sensitivity data are located in the directories Dice/data/sensitivities/HEU and Dice/data/sensitivities/PU.

\subsection{EXAMPLE APPLICATION SYSTEM}

Because of the availability of sensitivity data files (SDFs) for ${ }^{233} \mathrm{U}$ experiments in the ICSBEP Handbook,

a ${ }^{233} \mathrm{U}$ system was selected to demonstrate the VIBE methods for selection of benchmark experiments that are similar to the application system. The example system, referred to as Application 1 in 
ORNL/TM-2008/196, is a $12.2 \mathrm{~cm}$ radius sphere of $220 \mathrm{~g} \mathrm{U}$ per liter uranyl nitrate solution with no excess acid. The uranium is $100 \mathrm{wt} \%{ }^{233} \mathrm{U}$. The solution sphere is reflected by $0.25 \mathrm{~cm}$ of SS304 and $2 \mathrm{~cm}$ of water. The EALF calculated for this application is $0.280 \mathrm{eV}$. The $k_{\text {eff }}$ calculated for this system is 1.0036 .

Sensitivity coefficients were computed for this system using TSUNAMI-3D from SCALE 6 using the SCALE 238-group ENDF/B-VII.0 cross-section library. The TSUNAMI-3D input file for this case is shown in Fig. 3. The input and sensitivity data for this example are located in Dice/plugins/vibe/test -application.

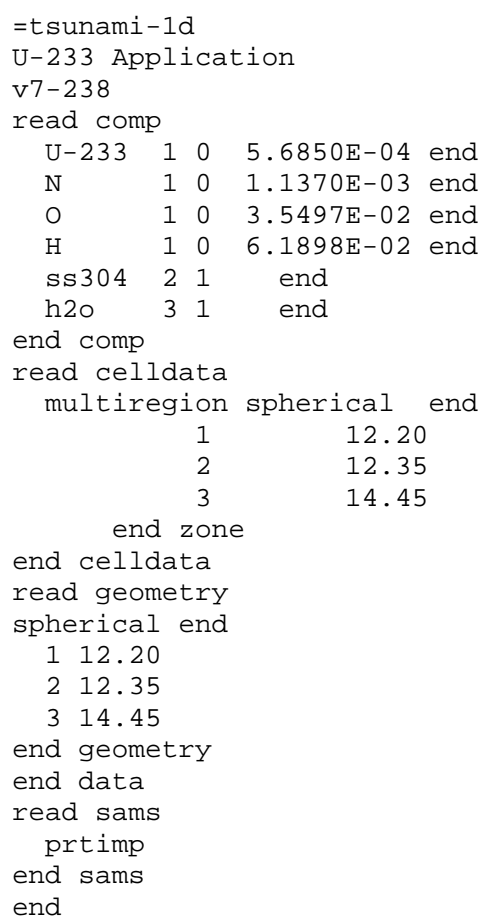

Fig. 3. TSUNAMI-1D input listing for ${ }^{233} \mathrm{U}$ application. 


\section{VIBE LAYOUT}

The VIBE tab of DICE is arranged into four sections: Sensitivity File Panel, Benchmark Set Panel, Benchmark List Panel, and Tables, as shown in Fig. 4. On initial viewing of VIBE, data will only be shown in the Sensitivity File Panel. The Sensitivity File Panel displays the locations of sensitivity data either distributed with the Handbook or available on the user's file system. The Benchmark Set Panel contains groupings of sensitivity data files that the user generates for further examination or export. The Benchmark List Panel provides a listing of the full directory path to the sensitivity data in the currently selected benchmark set. The Tables panel contains user-generated tables of group-collapsed sensitivity data or further information about the experiments in the benchmark set obtained from the DICE database. Guidance on how to display and use the data in each panel is provided in Section 5.

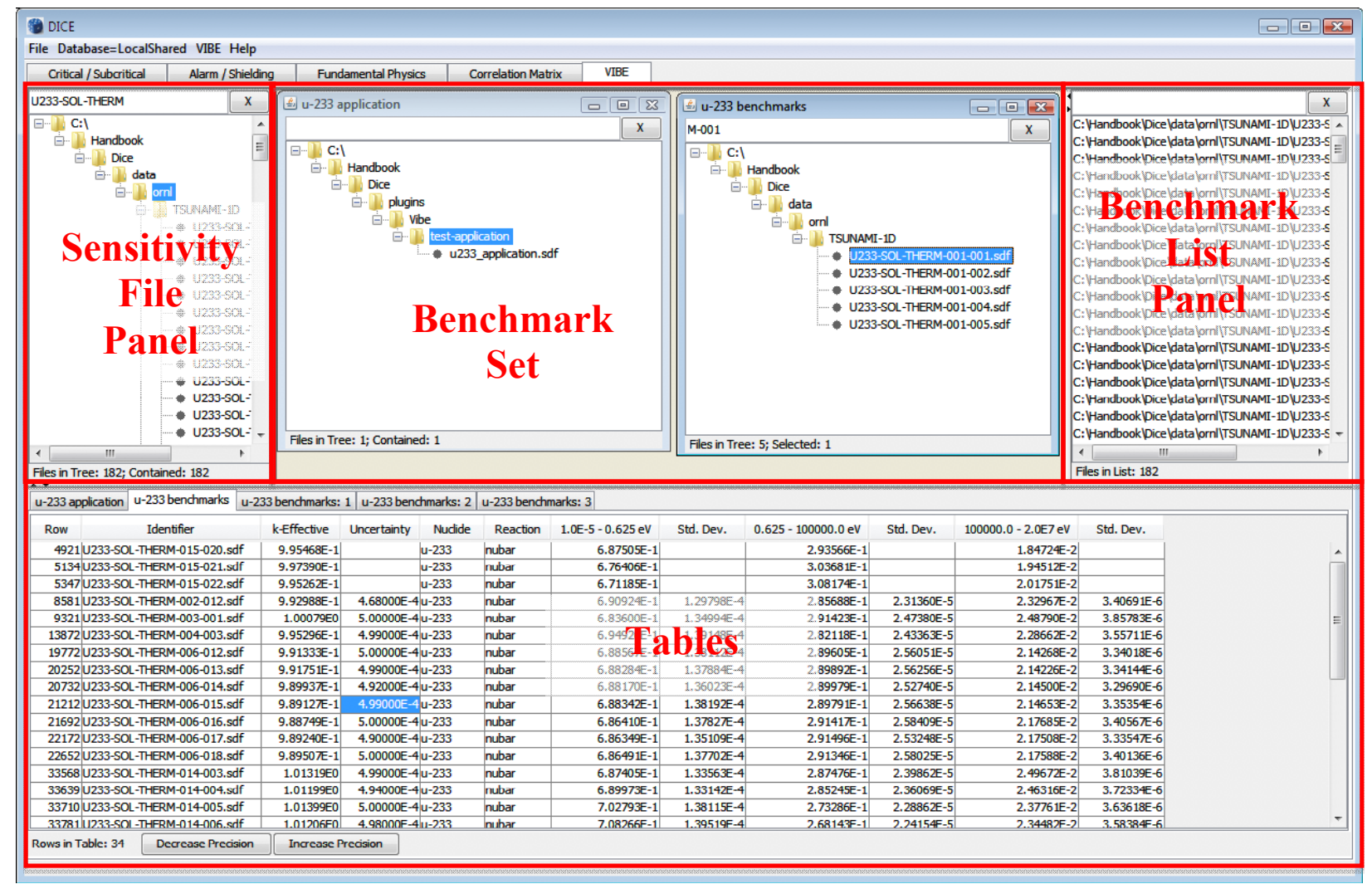

Fig. 4. Four sections of VIBE. 



\section{INTERPRETING SENSITIVITY DATA WITH VIBE}

The use of VIBE is demonstrated using the example ${ }^{233} \mathrm{U}$ application system described in Sect 3.2. After lauching DICE, begin by selecting the VIBE tab of DICE. VIBE will display an information message, as shown in Fig. 5. Here the user may view help information for VIBE or view instructions for installing the optional Javapeño plotting package of SCALE.

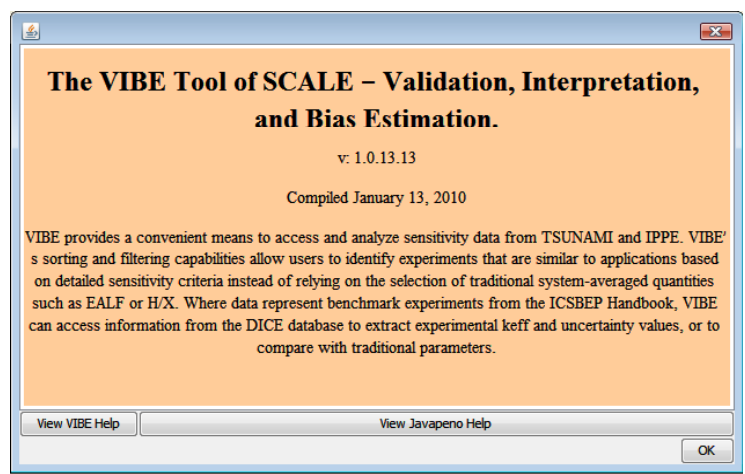

Fig. 5. VIBE information message.

VIBE will have the sensitivity data from the Handbook preloaded in the Sensitivity File Panel, as shown in Fig. 6.

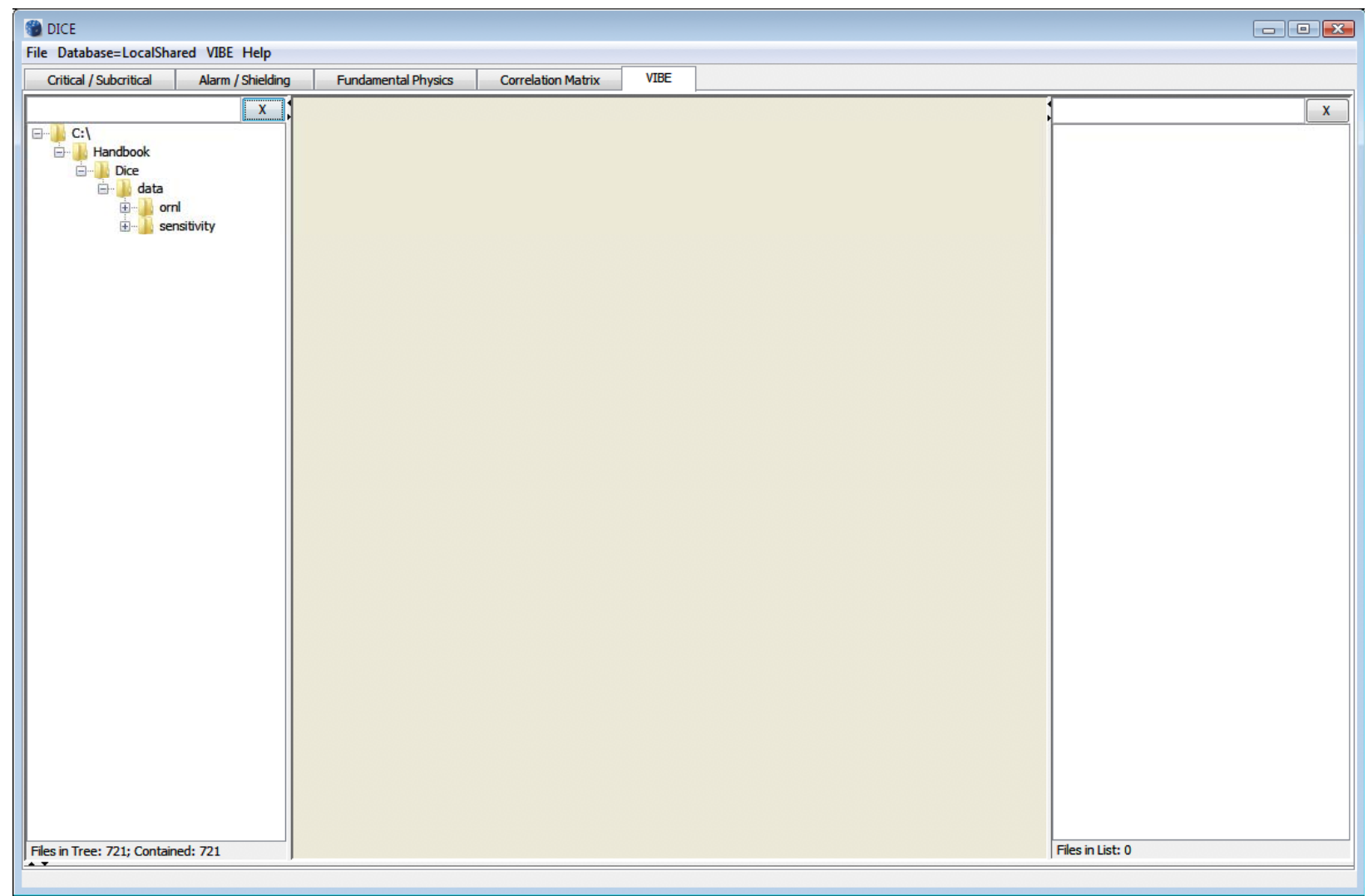

Fig. 6. VIBE tab of DICE. 
If the sensitivity data locations are not preloaded in the Sensitivity File Panel, or if the user desires to examine additional sensitivity data, the location of the sensitivity data can be identified. From the VIBE menu, select Add database location, shown in Fig. 7, and browse to the location of the directory containing the sensitivity data. The sensitivity data distributed with the Handbook are in the directory Dice/data.

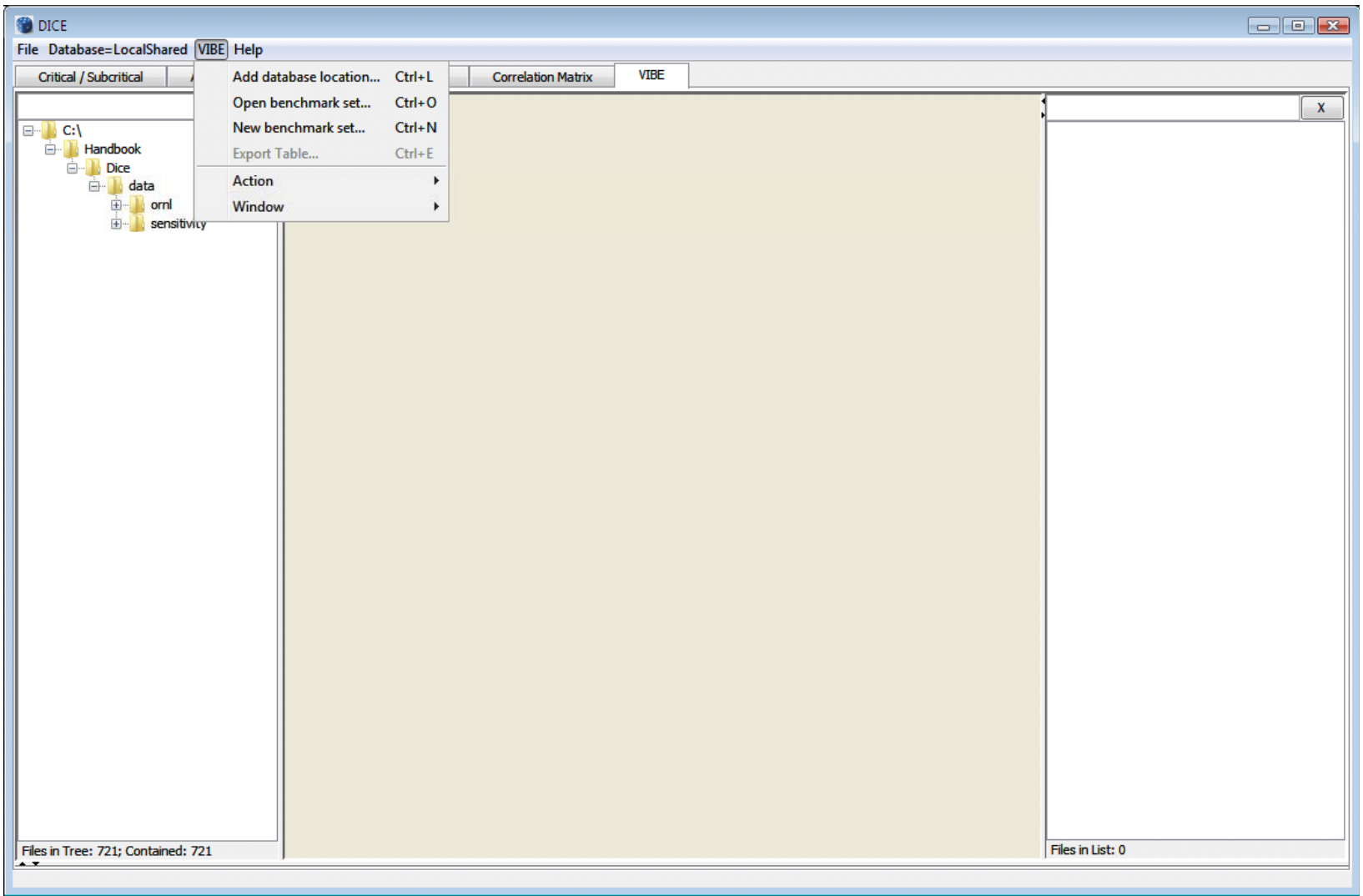

Fig. 7. VIBE with VIBE menu.

\subsection{QUANTIFICATION OF IMPORTANT PROCESSES IN THE APPLICATION}

The first step of identifying benchmarks that will exhibit the most similar biases as the application is to use the sensitivity data for the application to quantify the impact of important processes on its $k_{\text {eff }}$ value. The first step is to load the sensitivity data into VIBE by using the Select Database Location option of VIBE menu, as shown in Fig. 7, to mount Dice/plugins/vibe/test-application, as shown in Fig. 8. To begin examining the sensitivity of the application system, browse the file tree of sensitivity data to locate the sample application sensitivity data Dice/plugins/vibe/test application/u233_application.sdf, as shown in Fig. 9. Note that the number of files loaded in the Sensitivity File Panel and the number of files currently selected are shown at the bottom left corner of the application. 


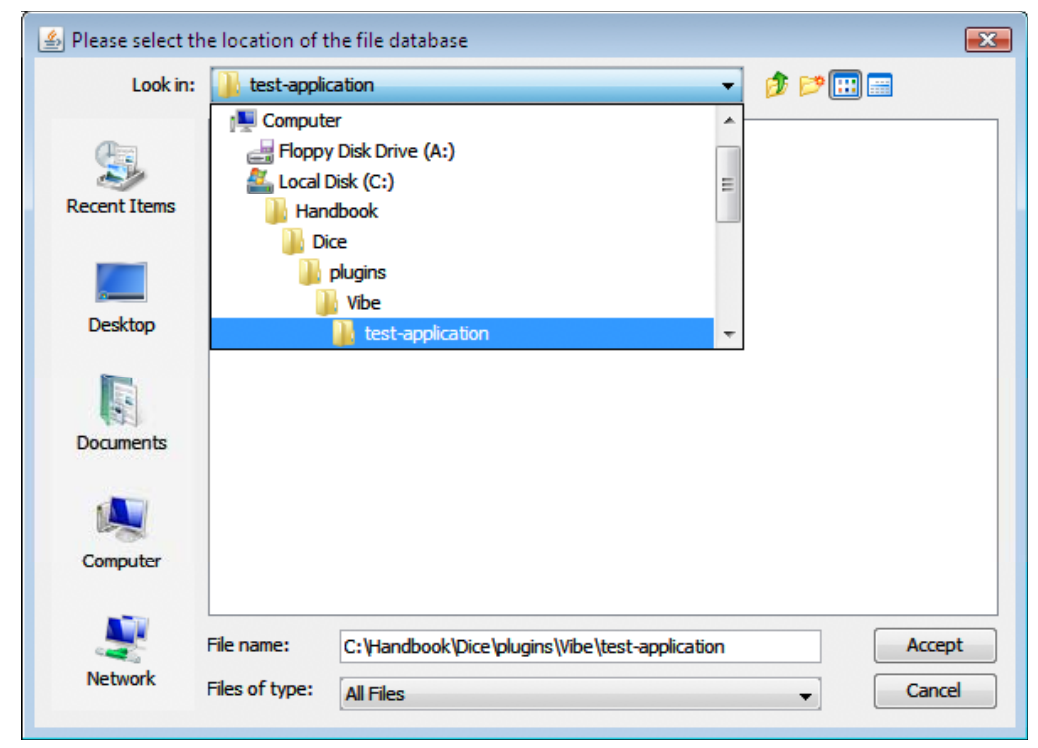

Fig. 8. File chooser with of ${ }^{233} \mathrm{U}$ application.

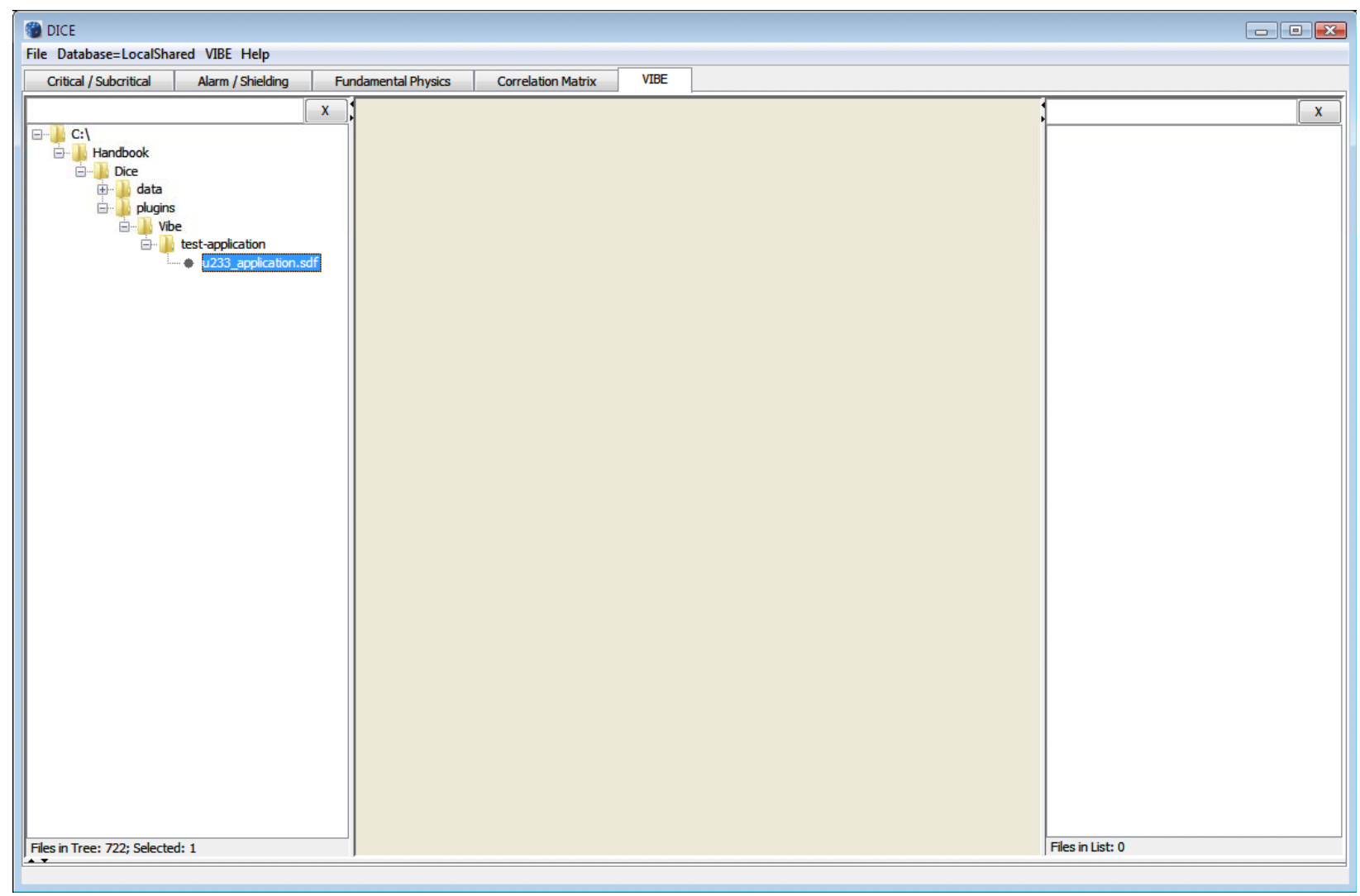

Fig. 9. Location of ${ }^{233} \mathrm{U}$ application in VIBE. 
A right-click on the SDF will open a pop-up menu with some options, as shown in Fig. 10. If the SCALE 6 data-plotting package Javapeño is installed, the option to Open in Javapeno will be available. If Javapeño is not installed, this step can be skipped. Javapeño is available as a free download from the SCALE website at http://www.ornl.gov/scale/javapeno.

If Javapeño is available, right-click on u233_application.sdf and select Open in Javapeno. It may take several seconds for the Javapeño application to launch with the SDF loaded. Because Javapeño operates with separate windows, the VIBE application will still be visible after Javapeño loads, as shown in Fig. 11.

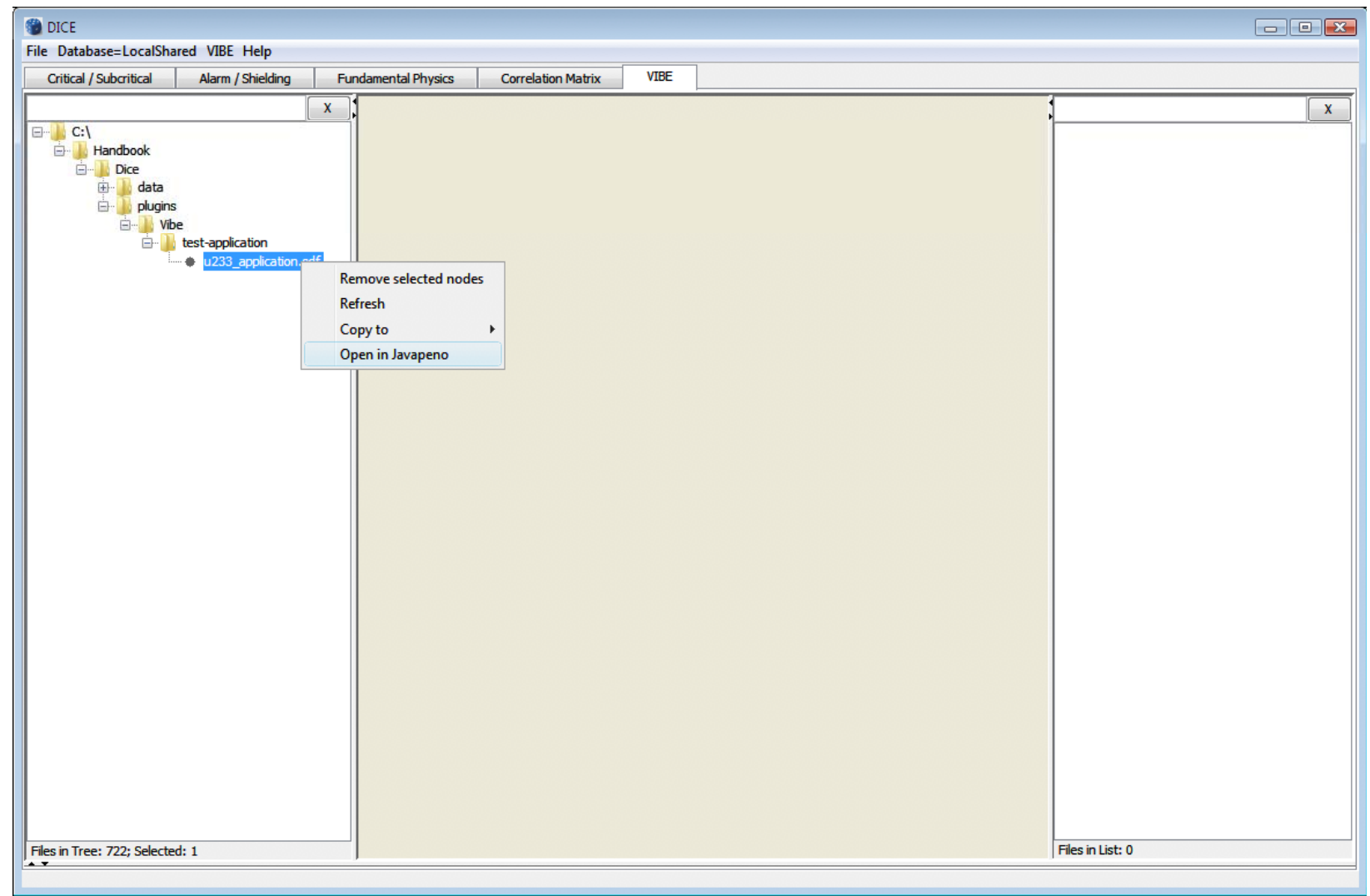

Fig. 10. VIBE application with SDF pop-up menu. 


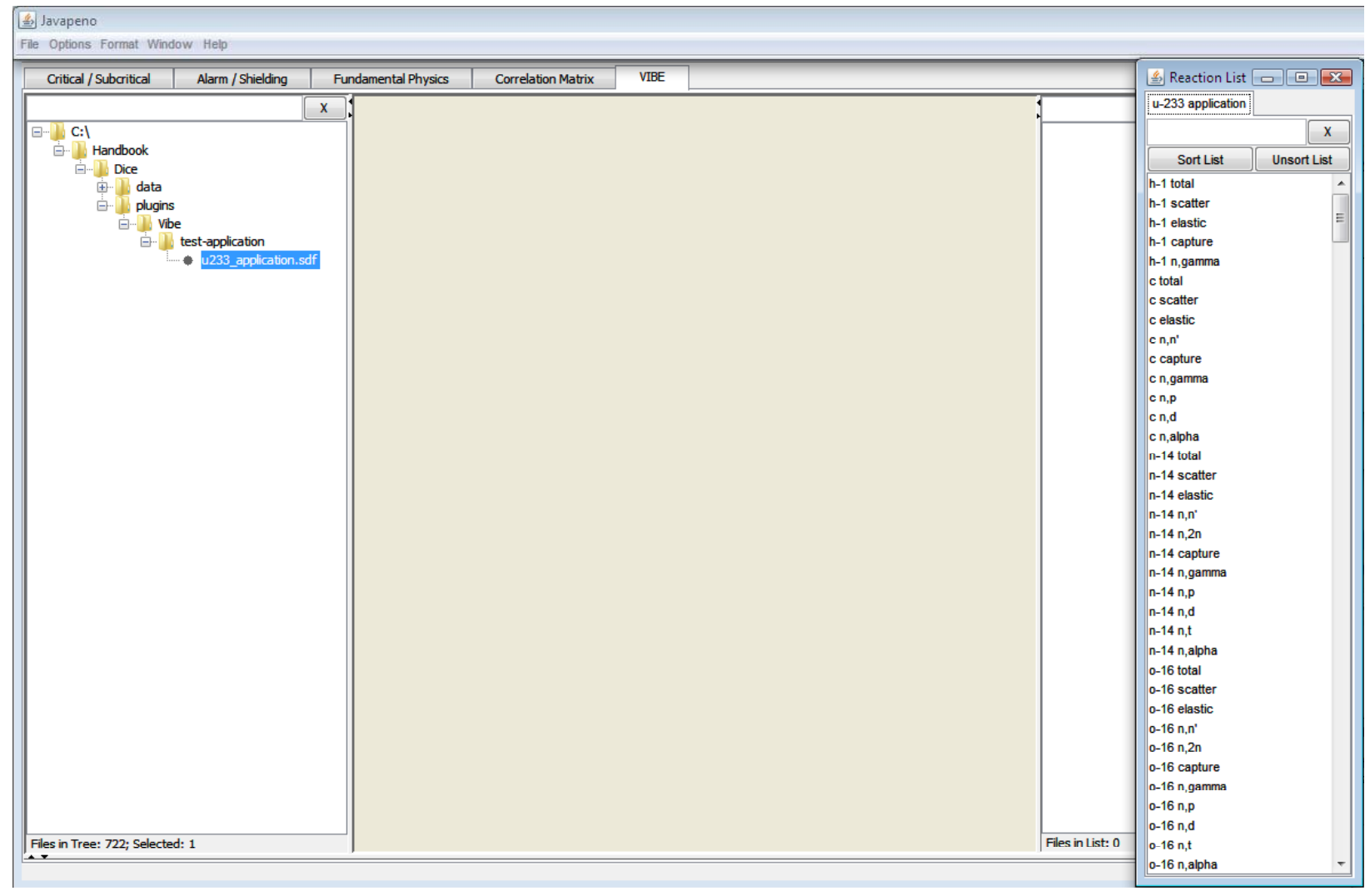

Fig. 11. View of Javapeño loaded over VIBE.

Plots may be viewed by double-clicking on items in the Javapeño Reaction List on the right side of the screen, as shown in Fig. 11. Plots of the fission and n, gamma sensitivities for ${ }^{233} \mathrm{U}$ reveal a high sensitivity at thermal energies, as expected, and some sensitivities at intermediate energies, as shown in Fig. 12. 


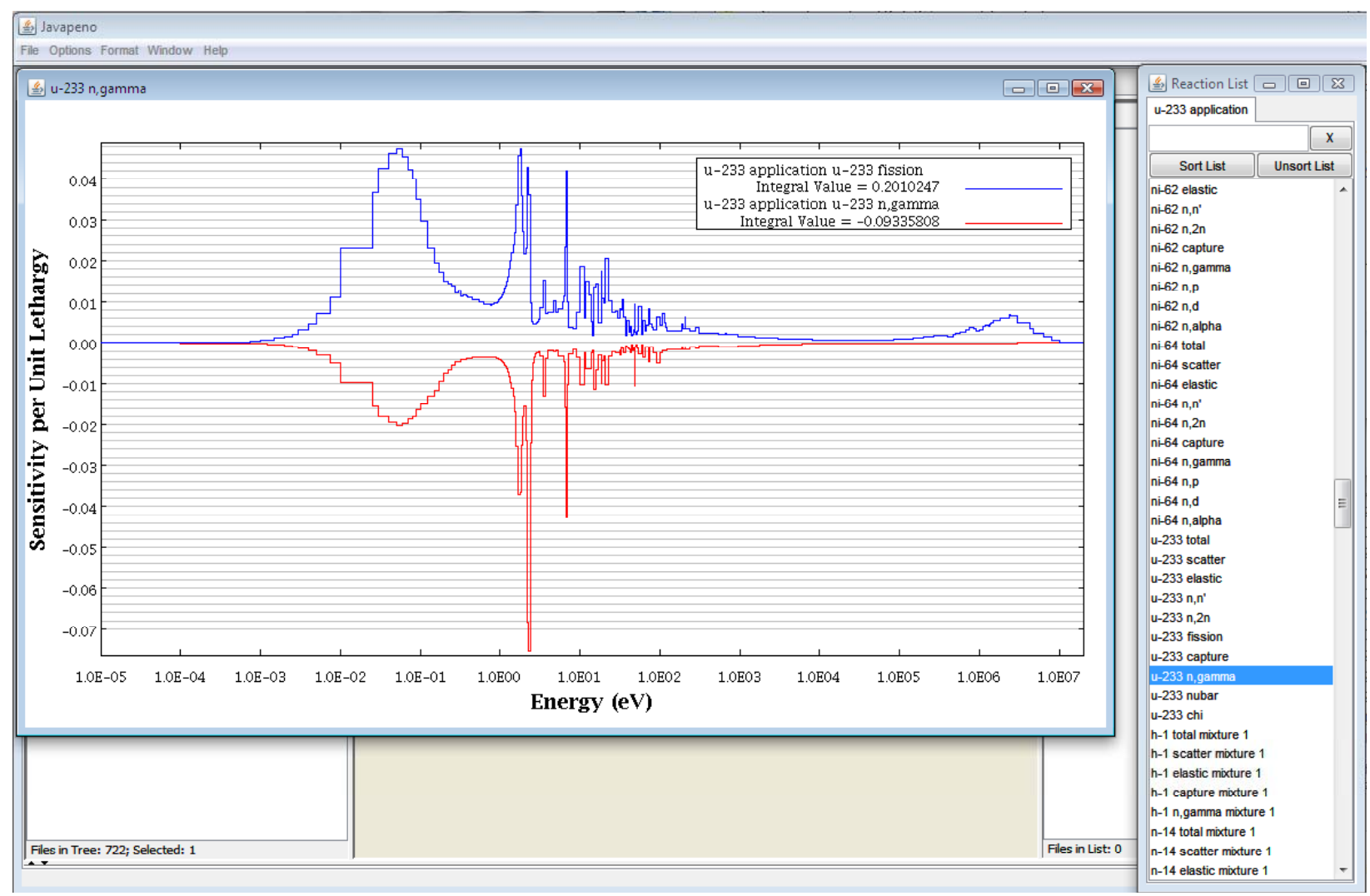

Fig. 12. Plotting ${ }^{233} \mathrm{U}$ fission and $\mathrm{n}$,gamma sensitivities for the ${ }^{233} \mathrm{U}$ application.

Performing a group collapse of the sensitivity data and applying VIBE's sorting and filtering capabilities will assist in identifying important processes in this system. To manipulate sensitivity data, the SDFs of interest must first be gathered in the VIBE benchmark set. For this example, the SDF for the ${ }^{233} \mathrm{U}$ application will be the only file in the set. VIBE provides two methods to start a new set. One is to rightclick on the SDF and select Copy to and then New Benchmark Set, as shown in Fig. 13. The other way is to simply drag and drop the SDF from the left pane to the center pane of VIBE. With either method, a dialog will appear requesting the name of the new set. Enter $u$-233 application and click OK, as shown in Fig. 14. 


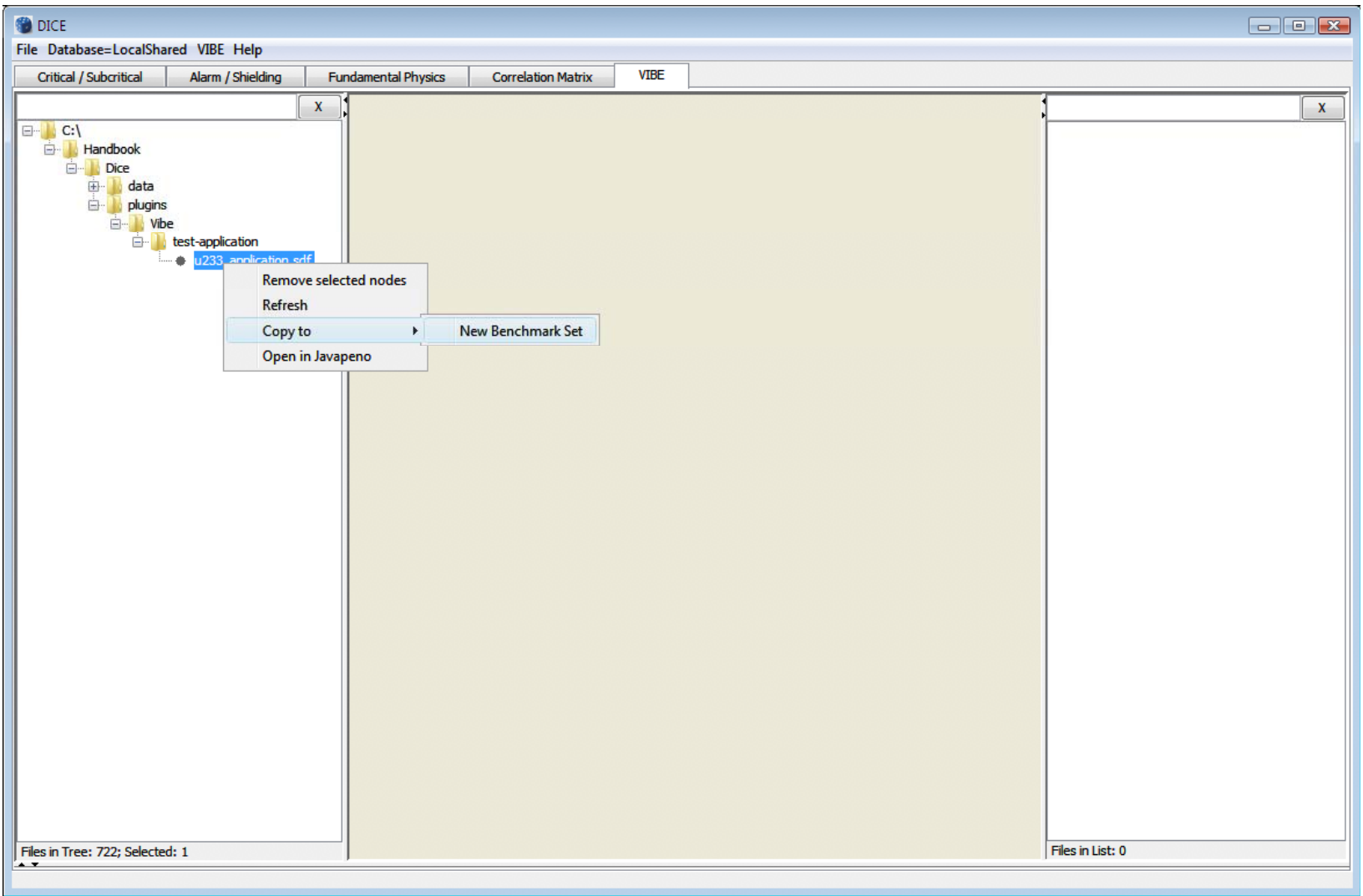

Fig. 13. Creating a new benchmark set in VIBE.

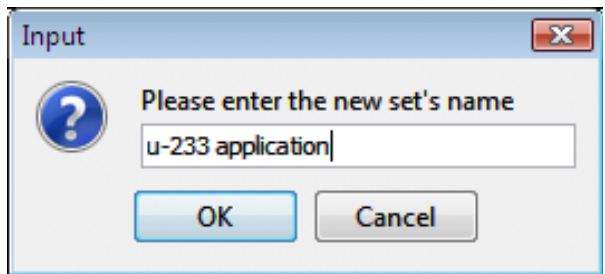

Fig. 14. Benchmark set name dialog.

At this point, VIBE will add a new window inside the center benchmark set pane, and the right list pane will show the full directory path to the SDF, as shown in Fig. 15. If more than one benchmark set is available, VIBE operations are conducted on the benchmark set in the currently selected benchmark set window. 


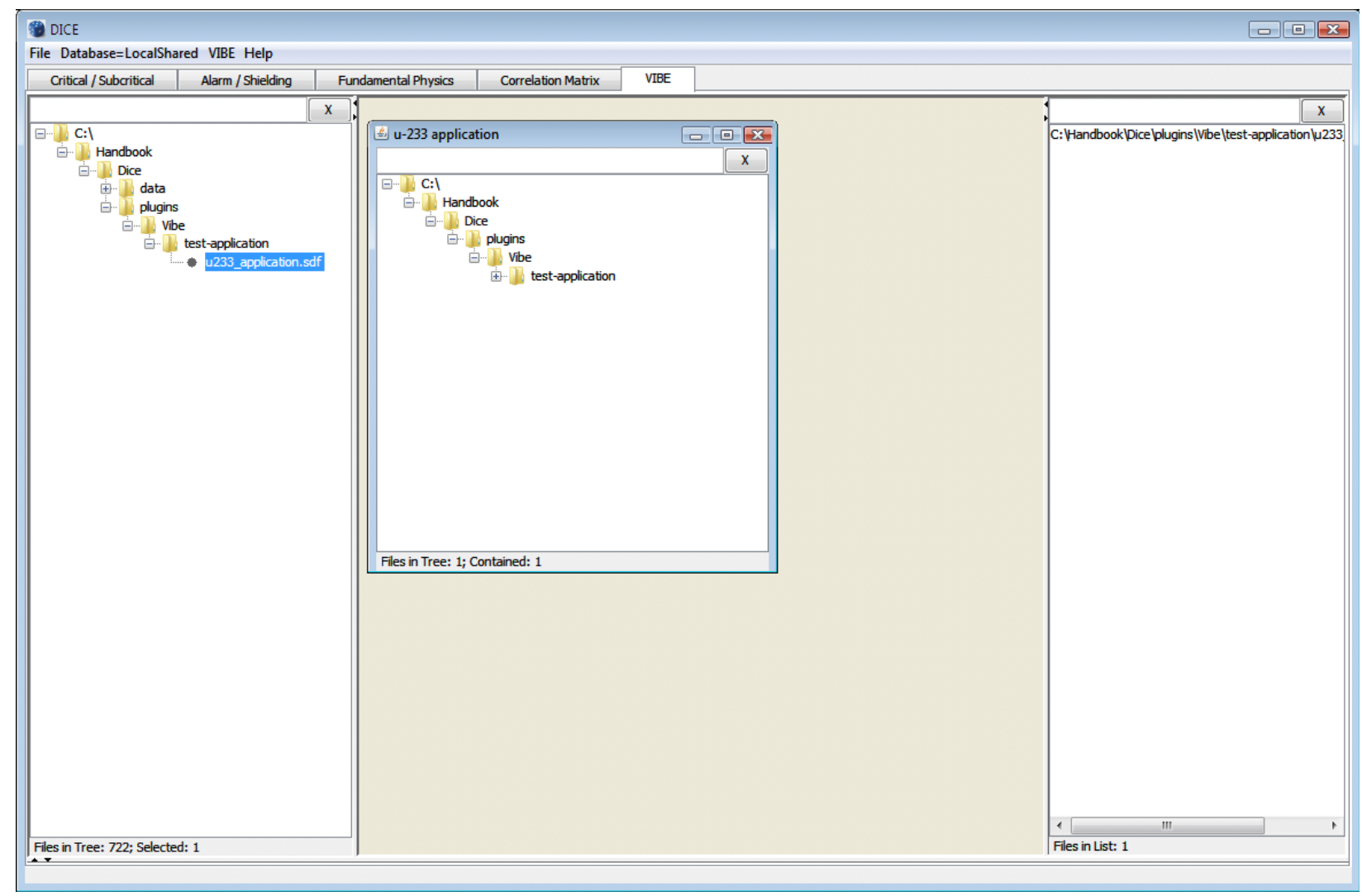

Fig. 15. VIBE with new benchmark set.

With a simple benchmark set established, a group collapse of the sensitivity data can be performed. Select Collapse Benchmark Set... from the VIBE Action menu, as shown in Fig. 16. Additionally, the collapse action can be performed from the benchmark set contextual menu by right-clicking in the benchmark set window, as shown in Fig. 17. In the Collapse Benchmark Set dialog, the boundaries for the group collapse can be selected by hand by clicking on the checkbox next to each desired boundary, or they can be set automatically using predefined group structures. The group structures available are 3-, 4-, 44-, and 238-group. By clicking the checkbox next to Use Predefined Structures, the 3-group structure, consistent with the ICSBEP 3-group boundaries, is selected. Other group structures can be selected from the drop-down menu, but in this example, the 3-group structure is used. Once this is selected, as shown in Fig. 18, click Collapse. 


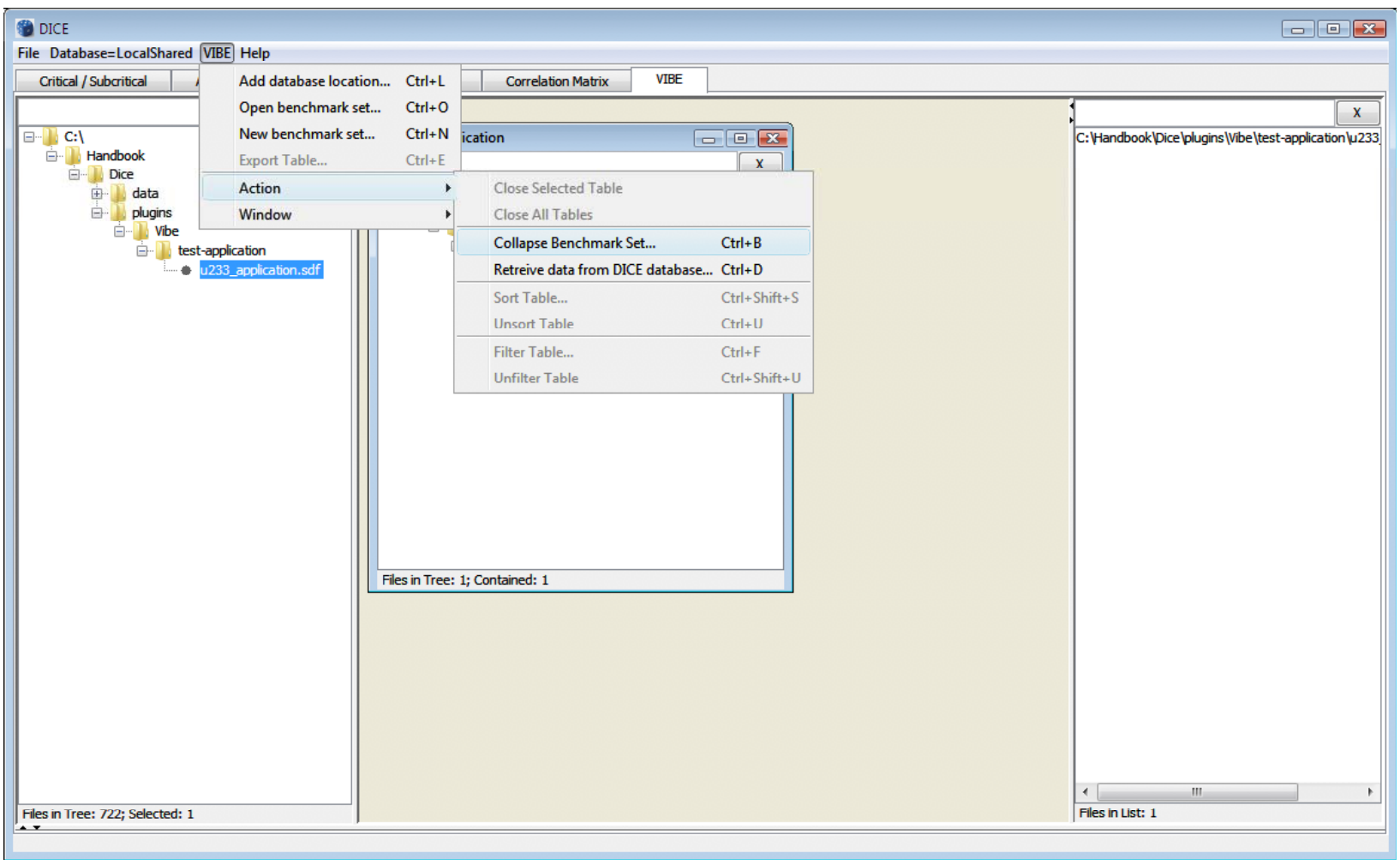

Fig. 16. VIBE action menu.

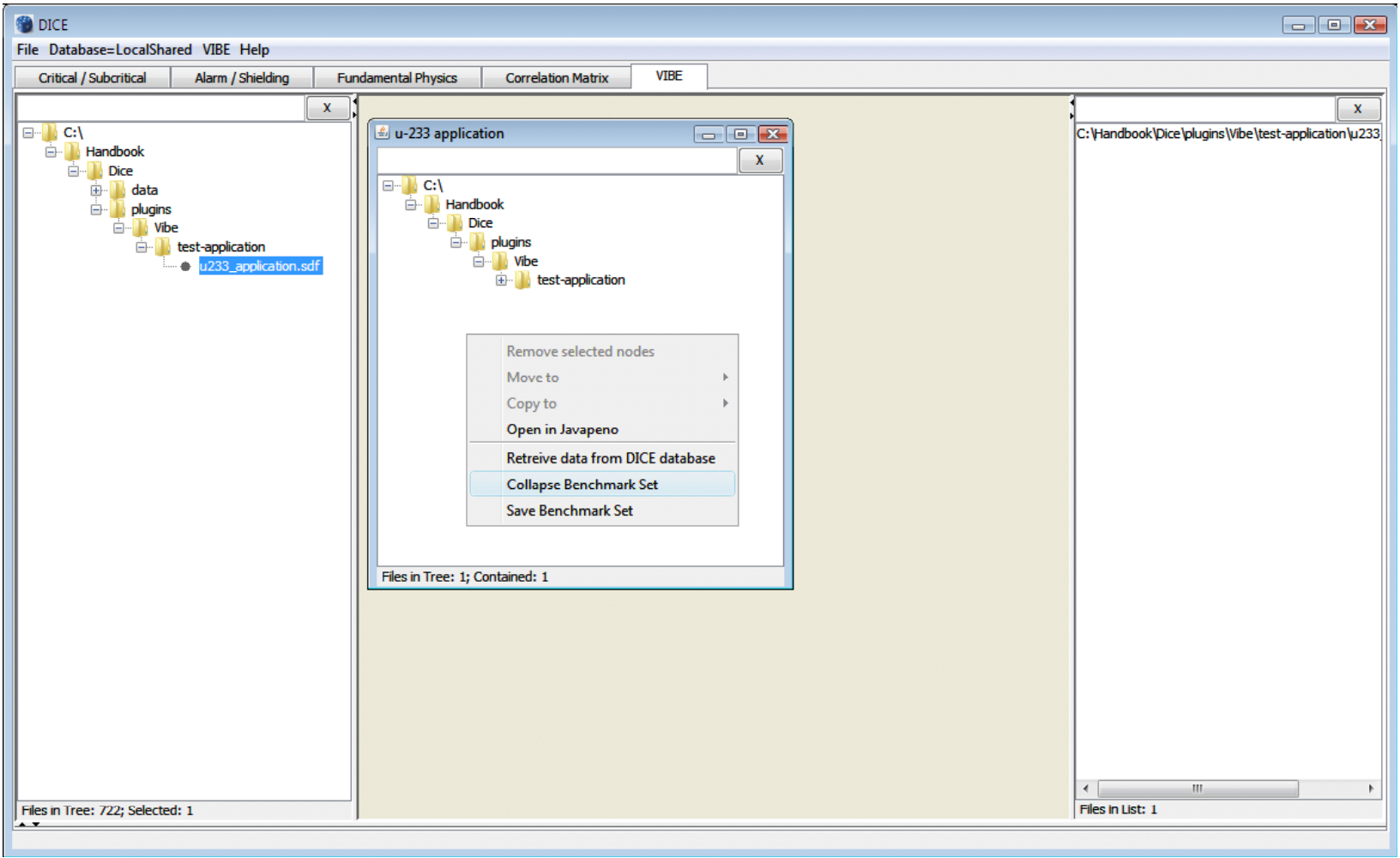

Fig. 17. VIBE contextual menu for benchmark sets. 


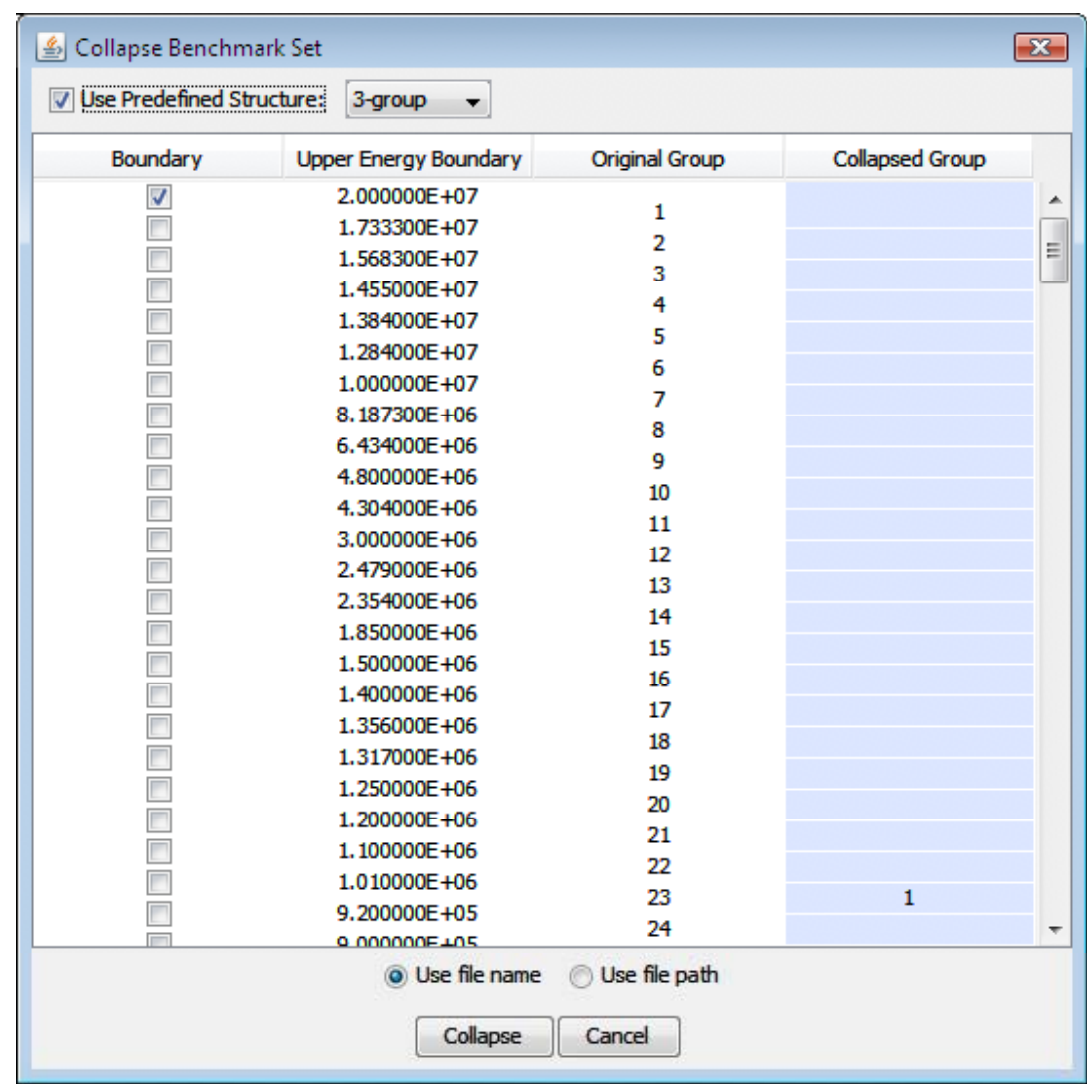

Fig. 18. VIBE group-collapsed dialog.

A new table will appear with the collapsed data, as shown in Fig. 19. The collapsed table contains columns for row number, SDF identifier, computed $k_{\text {eff }}$ and its uncertainty, nuclide and reaction for the sensitivity data, and 3-group sensitivity data and their uncertainties. The energy range for each collapsed group is shown at the top of the columns, and the statistical uncertainties due to Monte Carlo calculations are shown for each group in the column labeled Std. Dev. Note that the sensitivity data for this application was generated with the deterministic-based TSUNAMI-1D, so Monte Carlo uncertainties are not shown. The table contains a row for the nuclide-reaction pair for which sensitivity data are available on the SDF. For this file, there are 220 rows in the table, as shown in the lower left corner of the table pane. With so many rows, it may be difficult to visually inspect the data to identify the highest sensitivities for which validation data are desired. To simplify this process, the VIBE sorting capabilities will be used. 


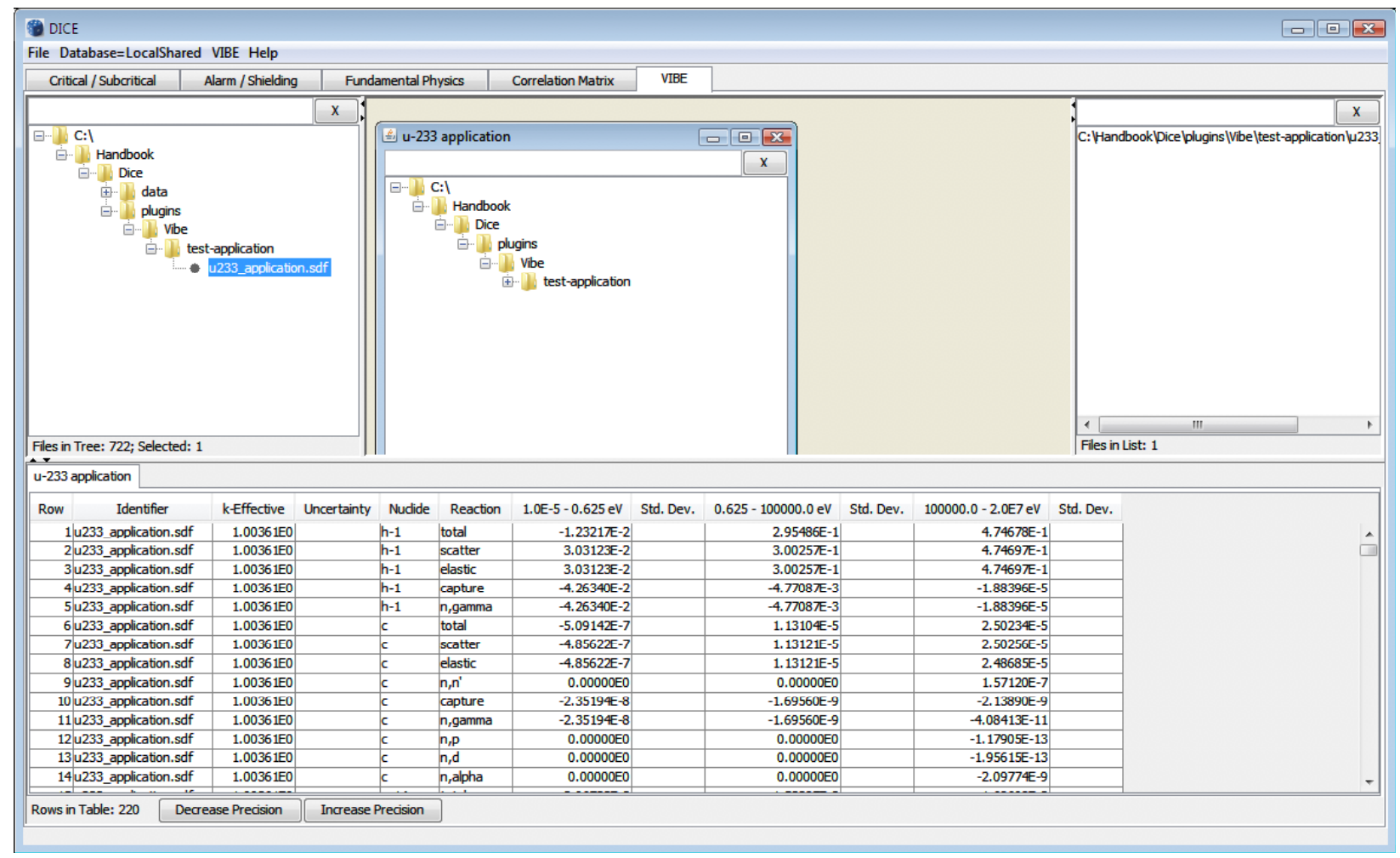

Fig. 19. VIBE with group-collapsed sensitivity data.

When a table is showing, the VIBE Action menu will present items to manipulate the currently selected table by closing, sorting, or filtering, as shown in Fig. 20. To identify nuclide-reaction pairs with high sensitivities, select Sort Table.... Begin by sorting the absolute values of the thermal data in descending order so that the sensitivities with the largest magnitude will be at the top of the table by setting the Sort dialog as shown in Fig. 21 and clicking Sort. Alternatively, the data can be sorted by clicking on any column header. An arrow will indicate if the data are sorted ascending or descending and vertical bars will be shown when the data are sorted by absolute value. 


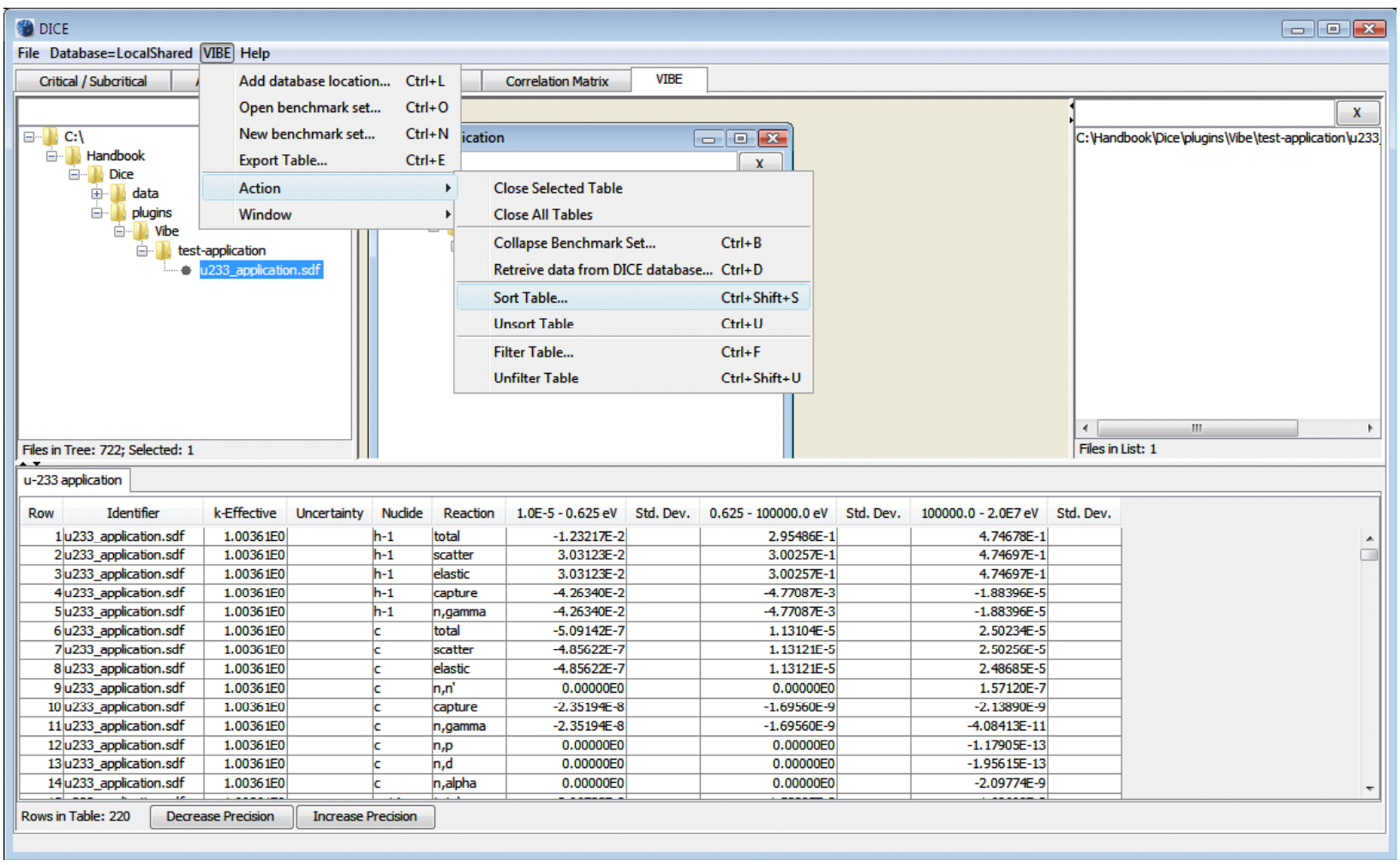

Fig. 20. VIBE action menu with table showing.

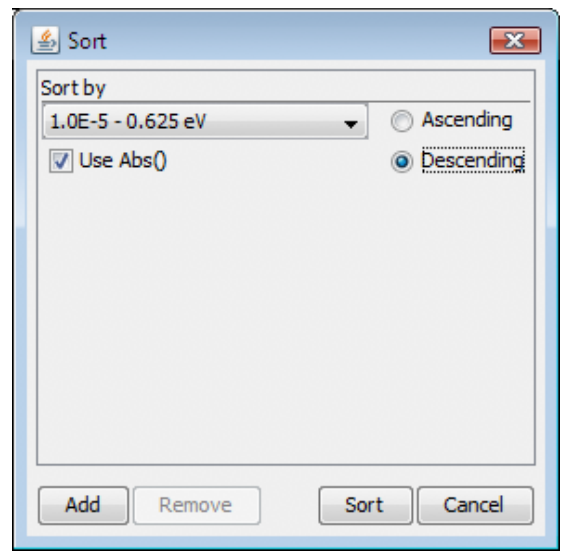

Fig. 21. VIBE sort dialog for sorting by thermal sensitivities by absolute in descending order.

The sorted sensitivities appear as shown in Fig. 22. As expected, the ${ }^{233} \mathrm{U}$ sensitivities have the highest values followed by ${ }^{1} \mathrm{H}$ and ${ }^{14} \mathrm{~N}$. Experiments with sensitivities similar to these will exhibit the most similar biases. An initial search will be conducted to identify experiments whose $k_{\text {eff }}$ sensitivity thermal ${ }^{233} \mathrm{U}$ nubar is near 0.69 . 


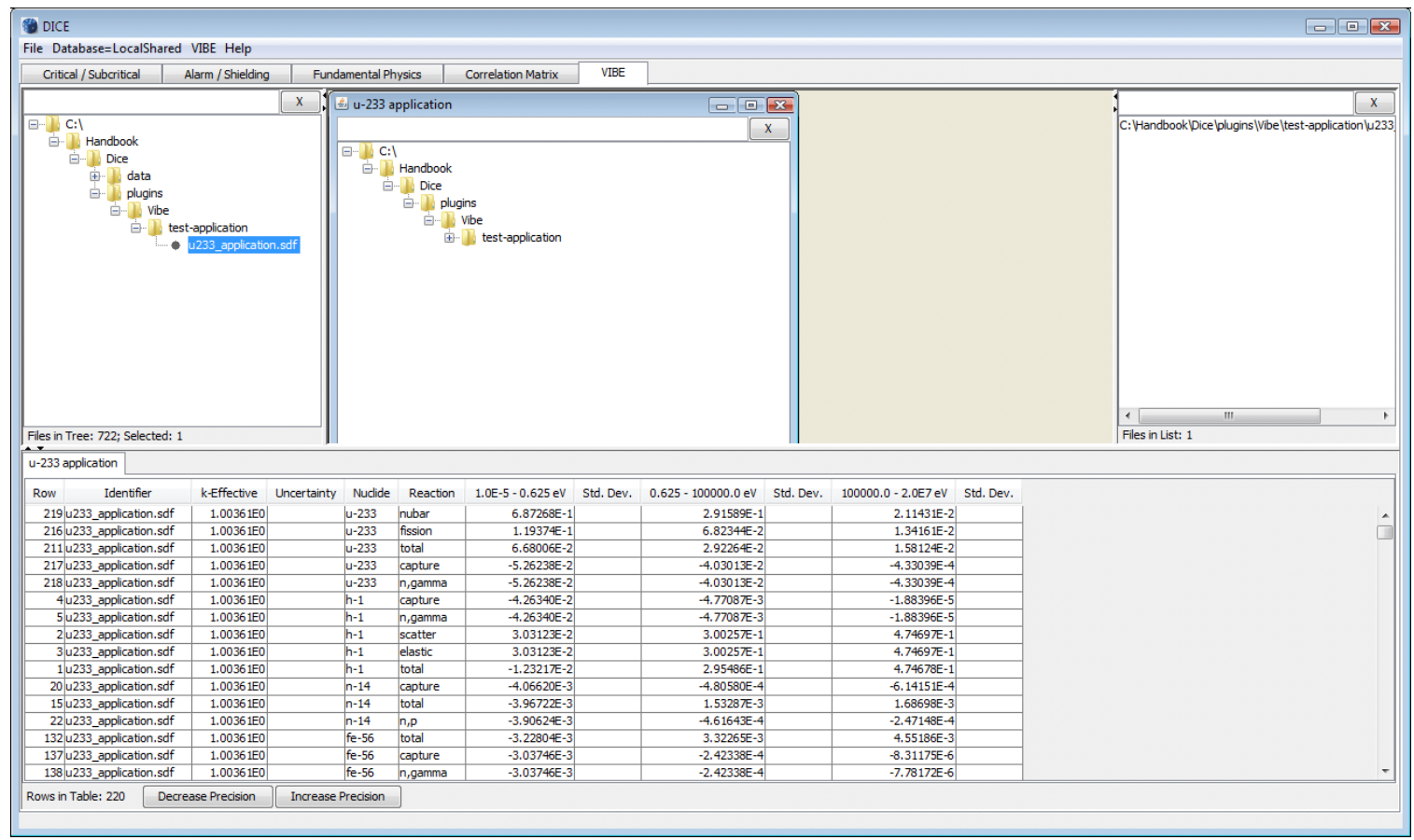

Fig. 22. VIBE with the absolute values of the thermal sensitivity data sorted in descending order.

\subsection{INITIAL SCREENING OF AVAILABLE EXPERIMENTS WITH VIBE}

VIBE provides a convenient means of down selecting from the hundreds of benchmarks with sensitivity data in the ICSBEP to the several that will be most useful for validation purposes. An initial screening can be conducted using the benchmark name and applying a filter to the file names. As this system is a thermal solution of ${ }^{233} \mathrm{U}$, it is expected that a U233-SOL-THERM system will provide the best match. Begin by typing U233-SOL - THERM in the filter field at the top of the Sensitivity File Panel, as shown in Fig. 23. As shown at the bottom of the panel, sensitivity data are available for 182 U233-SOL-THERM experiments. Some sensitivity data were generated with TSUNAMI-1D and some were generated with TSUNAMI-3D, but they are all suitable for comparison with the application.

Create a new benchmark set to examine the benchmark sensitivity data by dragging the ornl folder to the empty space in the center benchmark set pane, next to, but not in, the window called $u-233$ application, as shown in Fig. 24. A dialog will appear requesting the name of the new benchmark set; enter $u 233$ benchmarks, as shown in Fig. 25. 


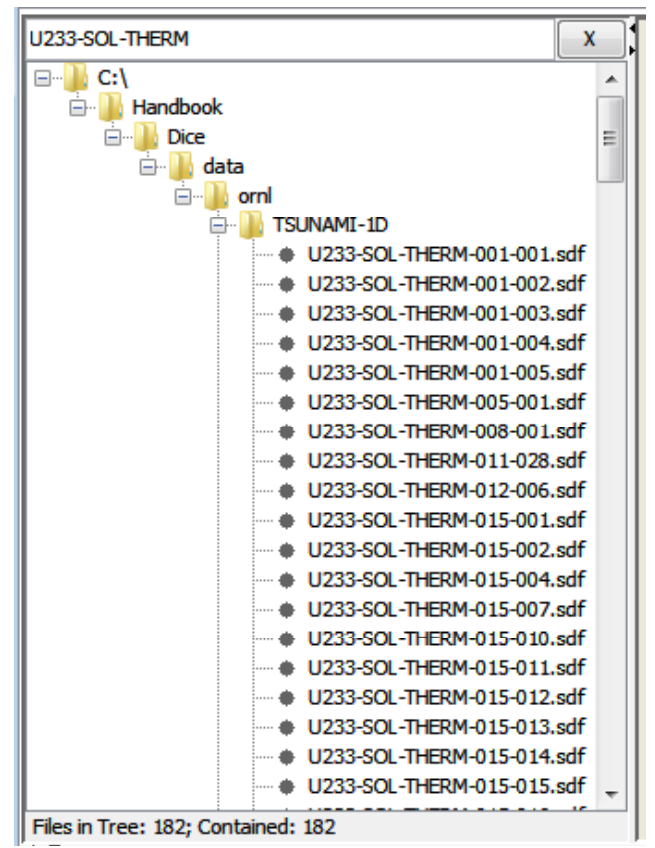

Fig. 23. VIBE Sensitivity File Panel with ICSBEP filter applied.

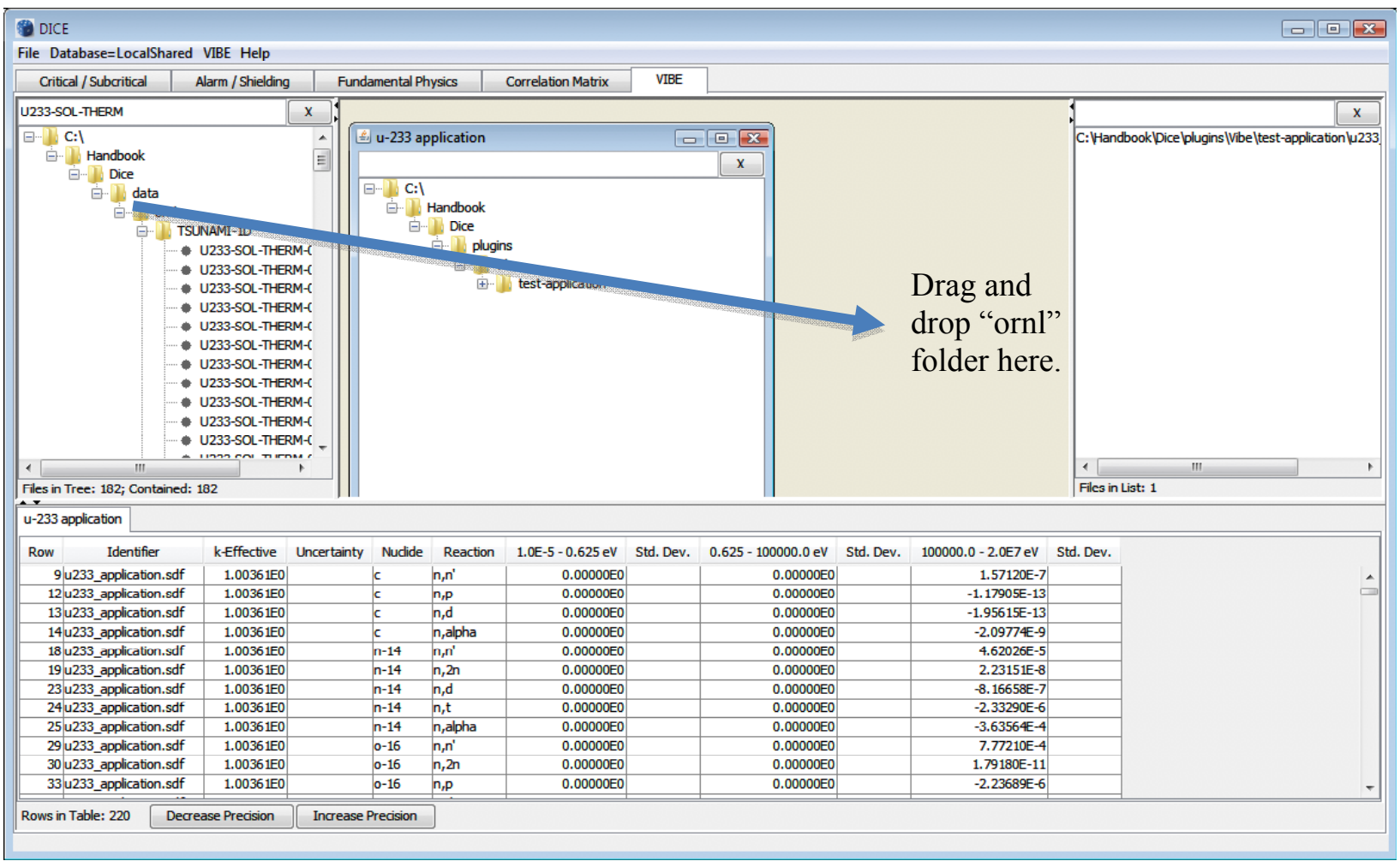

Fig. 24. Creation of new benchmark set for data distributed with ICSBEP Handbook. 


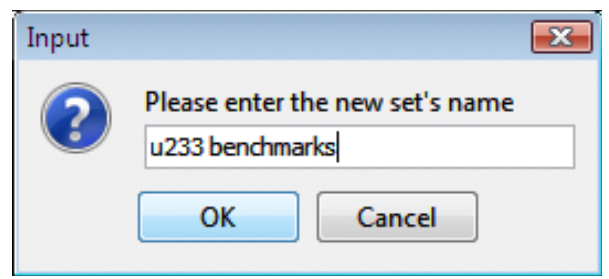

Fig. 25. VIBE benchmark set dialog for ${ }^{233} \mathrm{U}$ data.

Collapsing the sensitivity data to the same group structure as the application will allow identification of experiments with sensitivities, and thus computational biases, similar to those of the application. To perform a group collapse on all experiments in the $\mathrm{u} 233$ benchmarks set, repeat the same process as was previously performed for the u-233 application set. With the u233 benchmarks benchmark set window selected, select Collapse Benchmark Set from the Action menu, in the Collapse Benchmark Set dialog, check the box to use the predefined 3-group structure, and click Collapse. The collapse of the 182 files from the handbook DVD may take several seconds to complete. The procedure will likely occur faster if the SDFs from the handbook are first copied to a local hard drive. The resulting collapsed data will appear as shown in Fig. 26. Note at the lower left corner of the table that the status line states there are 49,221 rows in this collapsed table. The number of rows corresponds to the number of sensitivity profiles in all $182 \mathrm{SDFs}$ considered. Note that the initial order of the table corresponds to the order of the SDFs in the benchmark set and the order of the profiles within each SDF.

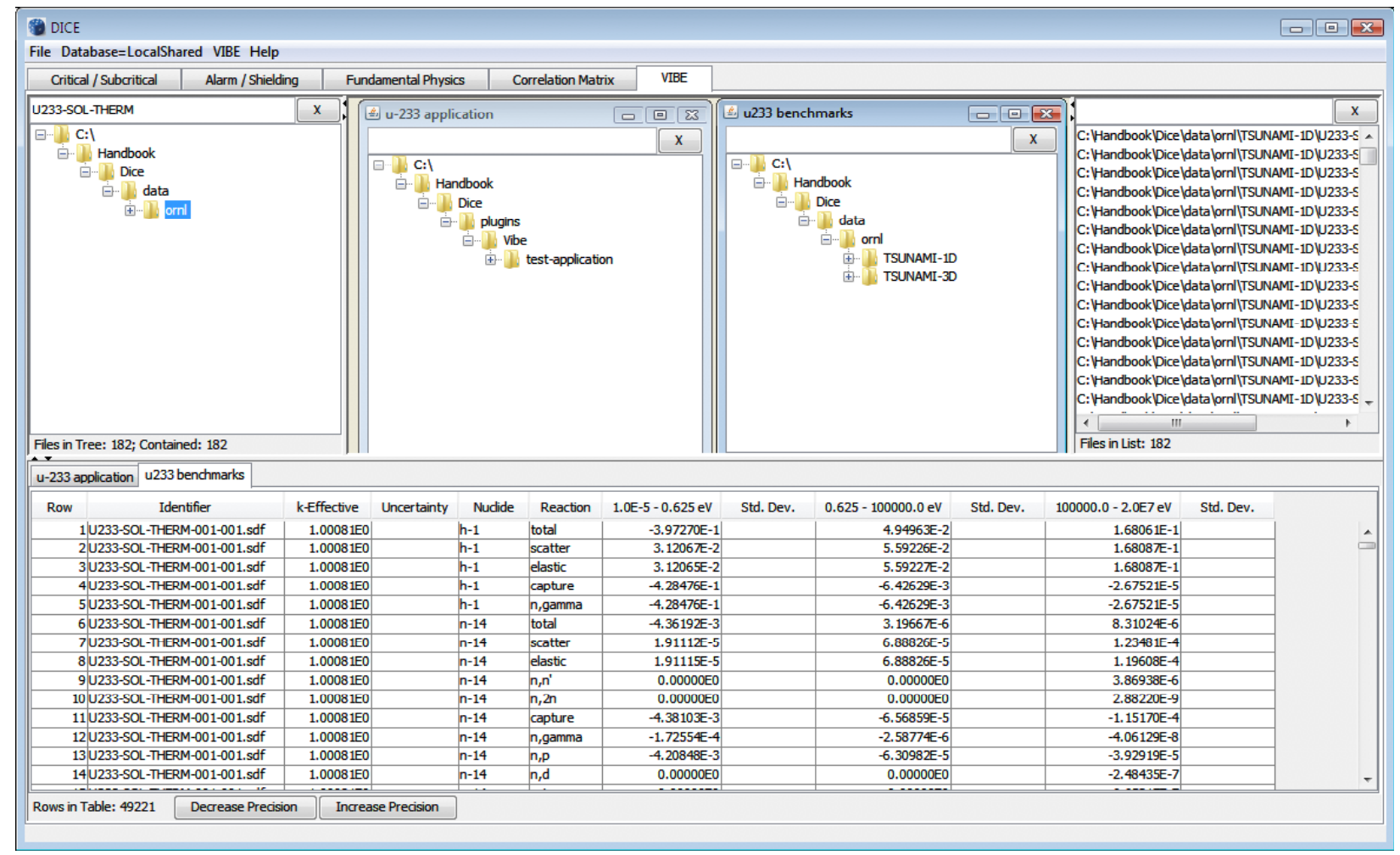

Fig. 26. VIBE with group-collapsed sensitivity data for benchmarks from the ICSBEP Handbook. 
To examine the availability of sensitivities in the benchmarks that are similar to those in the application, VIBE's sorting and filtering capabilities will be used. As shown in Fig. 22, the application is most sensitive to ${ }^{233} \mathrm{U}$ nubar in the thermal range. Begin by filtering the benchmarks to eliminate all other reactions except ${ }^{233} \mathrm{U}$ nubar. With the $\mathrm{u} 233$ benchmarks table showing, select Filter Table... from the VIBE Action menu, as shown in Fig. 27. In the Filter dialog, select Nuclide from the first pull-down menu, select is from the second pull-down menu, and type u-233 in the specification field. Next, click Add Constraint, and set the next constraint for reaction is nubar. Ensure that All of These is selected at the bottom of the dialog so it appears as shown in Fig. 28, and then select Basic Filter to apply the filter to the table.

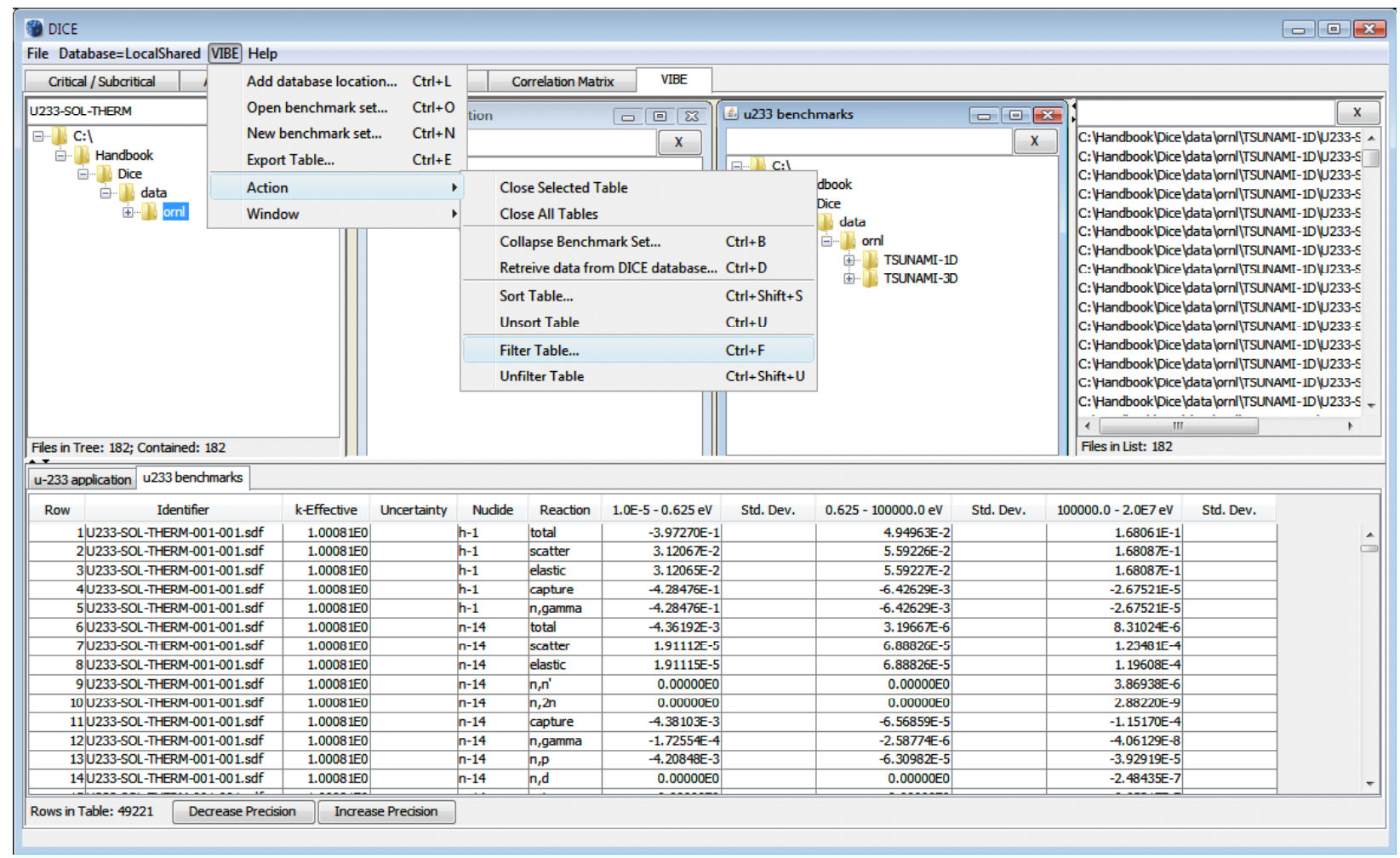

Fig. 27. VIBE action menu item for table filtering. 


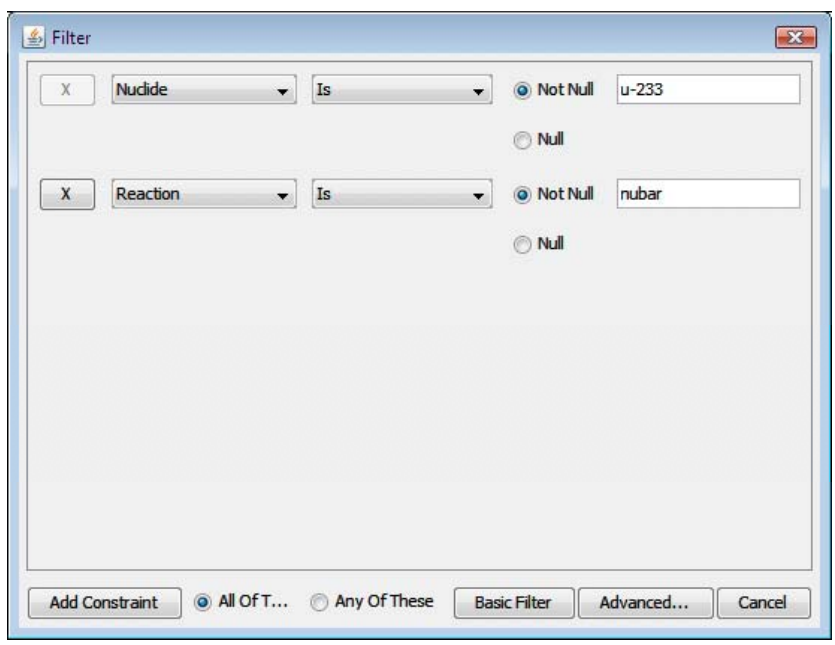

Fig. 28. VIBE filter dialog for ${ }^{233} \mathrm{U}$ nubar.

The filtered table will appear as shown in Fig. 29. Note that there are now only 182 rows in the table. Sorting the table by the thermal sensitivity in descending order provides the results shown in Fig. 30. Note that the highest ${ }^{233} \mathrm{U}$ nubar thermal sensitivities are $>0.95$, where the application sensitivity is 0.69 , as shown in Fig. 22. Although high coverage is provided by these benchmarks, the benchmarks with the highest thermal sensitivities may exaggerate the bias due to ${ }^{233} \mathrm{U}$ at thermal energies, while underrepresenting important processes at other energies or in other nuclides and reducing similarity to the application system. Scrolling down through the table, it can be observed that there are many benchmarks with ${ }^{233} \mathrm{U}$ nubar thermal sensitivities near 0.69 , as shown in Fig. 31. The number of benchmarks with sensitivities near 0.69 can be determined using VIBE's filter capabilities. 


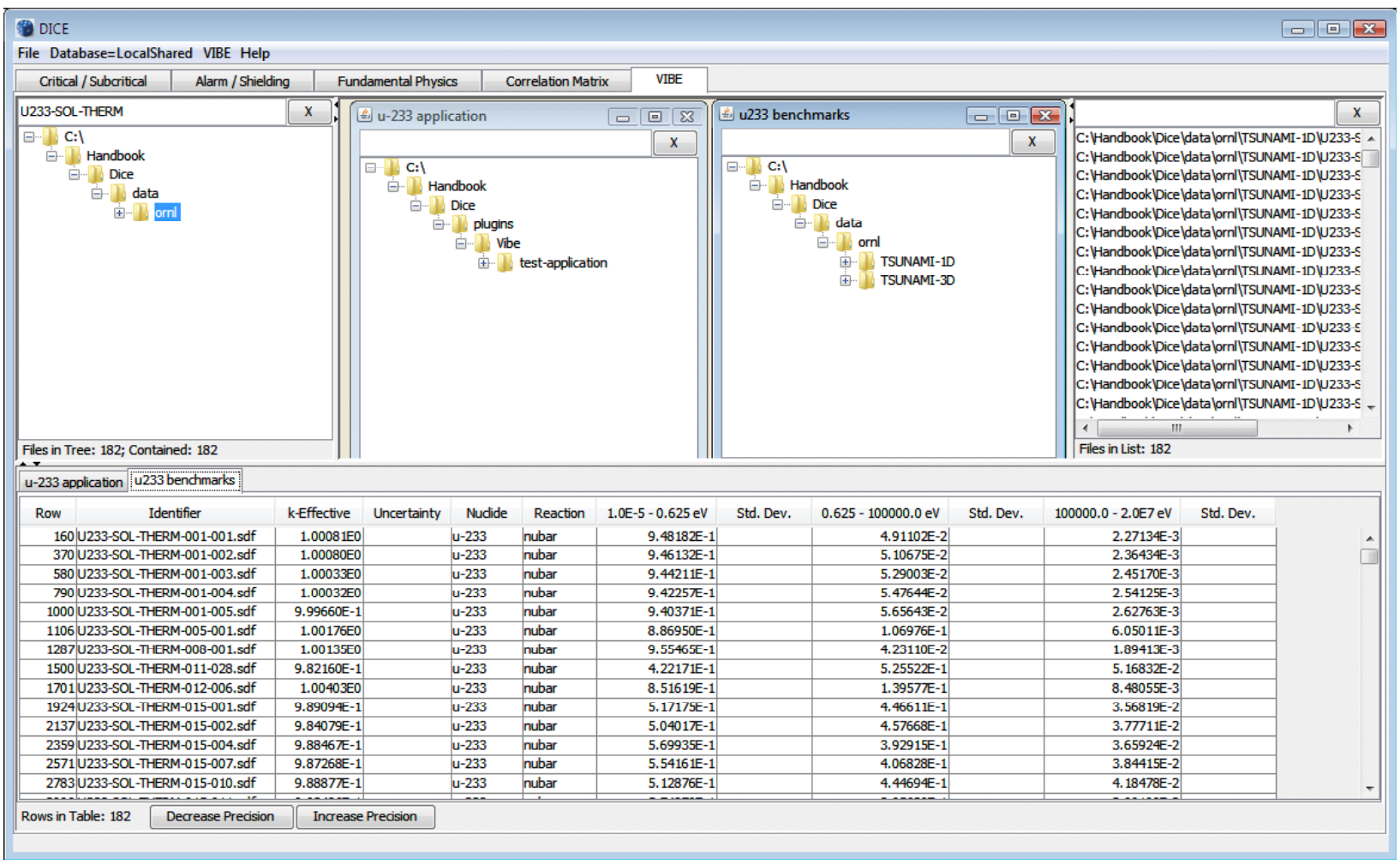

Fig. 29. VIBE with ${ }^{233} \mathrm{U}$ filter applied to ICSBEP Handbook data.

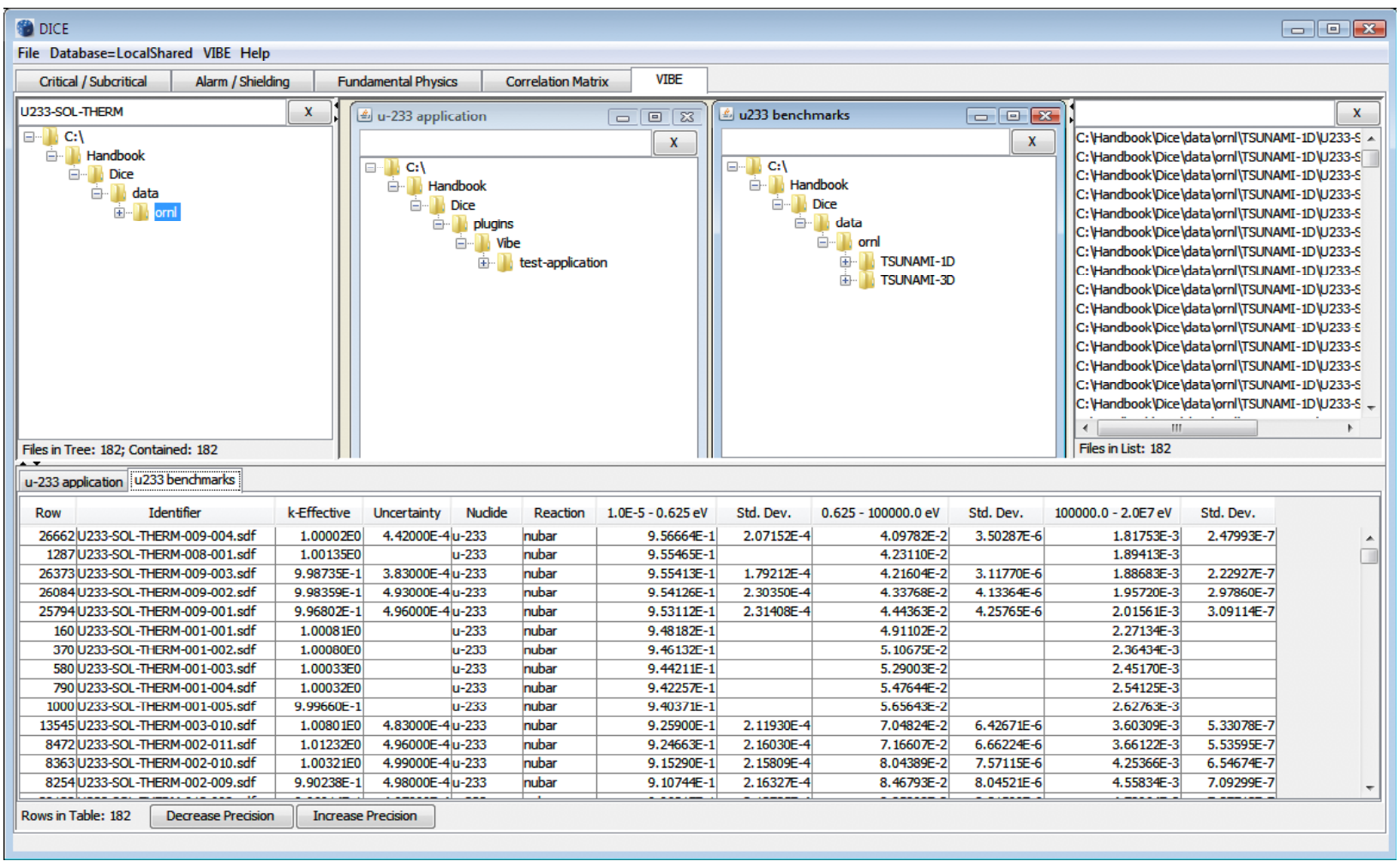

Fig. 30. VIBE with ${ }^{233} \mathrm{U}$ nubar filter and descending sorting for thermal sensitivities applied to ICSBEP Handbook data. 


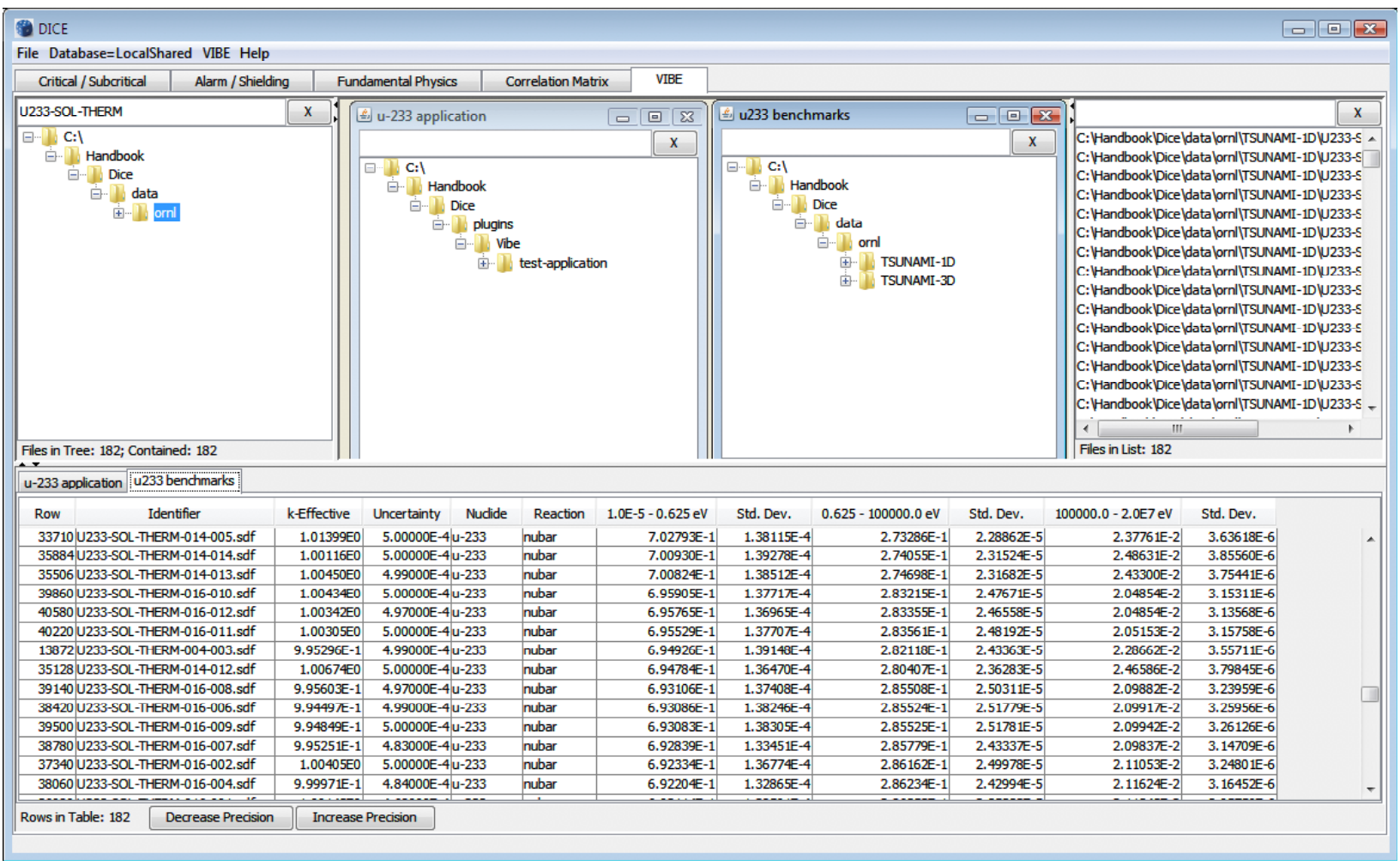

Fig. 31. Values near 0.69 from VIBE with ${ }^{233} \mathrm{U}$ filter and descending sorting for thermal sensitivities applied to ICSBEP Handbook data.

Returning to the filter dialog, available from the Action menu, add additional constraints to show only systems with ${ }^{233} \mathrm{U}$ nubar sensitivities with values between 0.67 and 0.71 , inclusive, as shown in Fig. 32 . Once the filter is applied, the number of matching benchmarks is 34, as shown in Fig. 33.

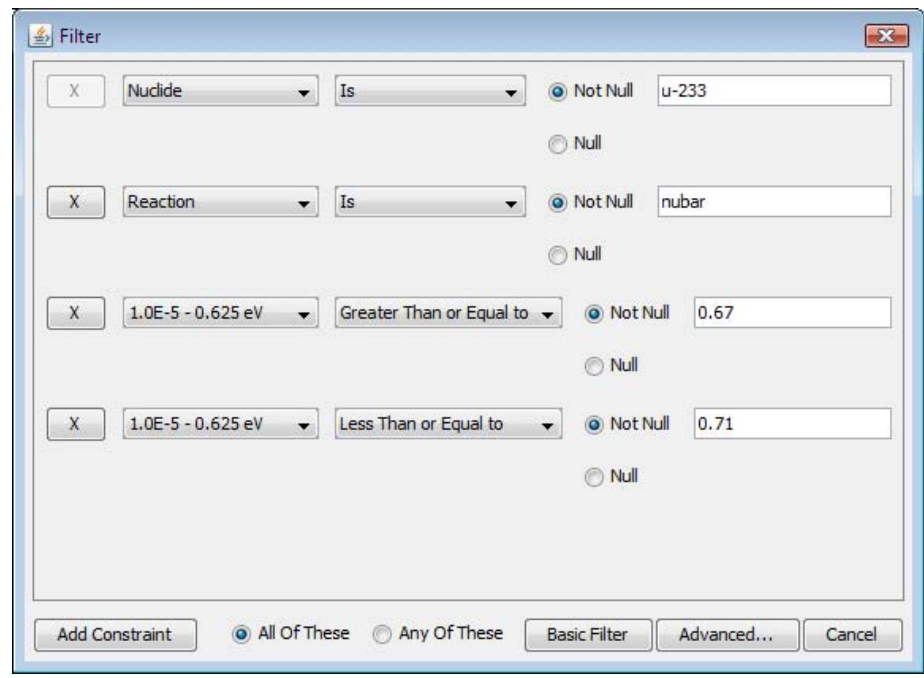

Fig. 32. VIBE Filter dialog for thermal ${ }^{233} \mathrm{U}$ nubar sensitivities with values $\geq 0.67$ and $\leq 0.71$. 


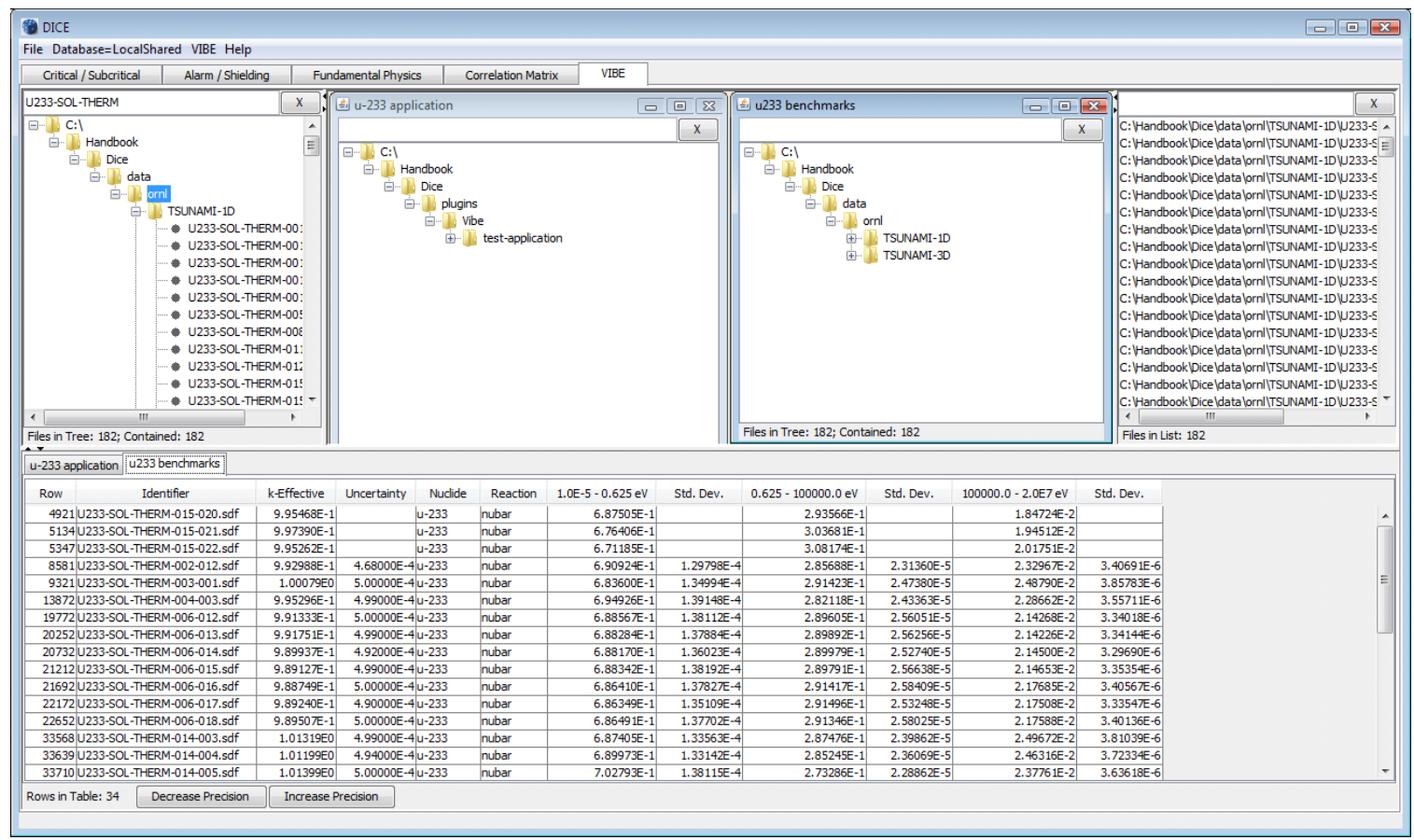

Fig. 33. VIBE with ${ }^{233} \mathrm{U}$ nubar thermal sensitivities near 0.69 filter applied to ${ }^{233} \mathrm{U}$ benchmarks.

The energy-dependent sensitivity data for the benchmarks can be visually compared to the sensitivities of the application using Javapeño. To open the benchmark SDF in Javapeño for the first benchmark shown in the table, U233-SOL-THERM-016-003, type U233-SOL-THERM-016-003 in the filter field of the u233 benchmarks benchmark set window in the center pane of VIBE, as shown in Fig. 34. Only the matching SDF will remain visible in the tree as long as the filter is applied. Right-click on the .sdf file name and select Open in Javapeno. After the file is read from the handbook DVD, a new tab containing the sensitivity data will be added to the Javapeño reaction list, as shown in Fig. 35. 


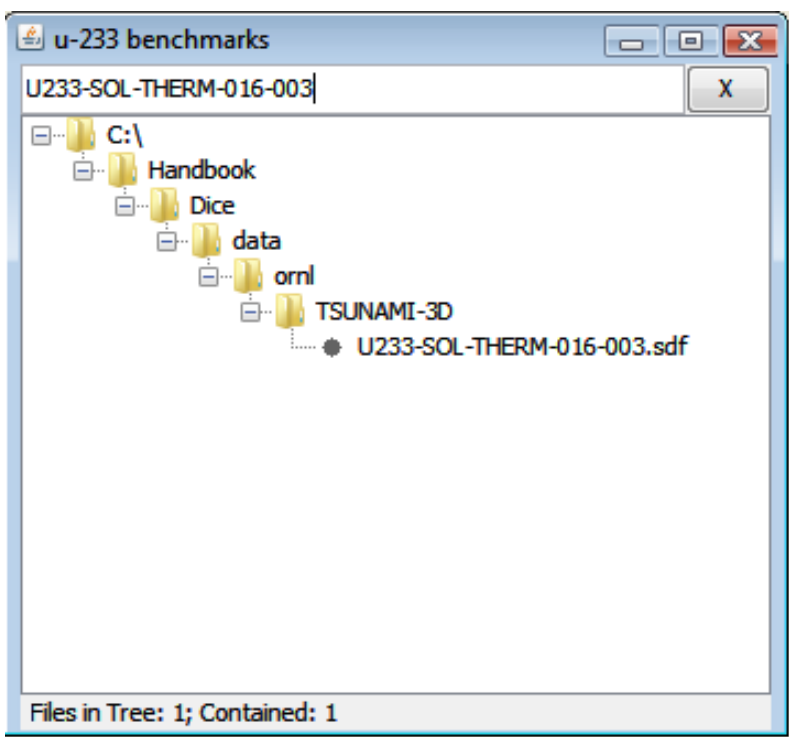

Fig. 34. Handbook benchmark set with filter applied.

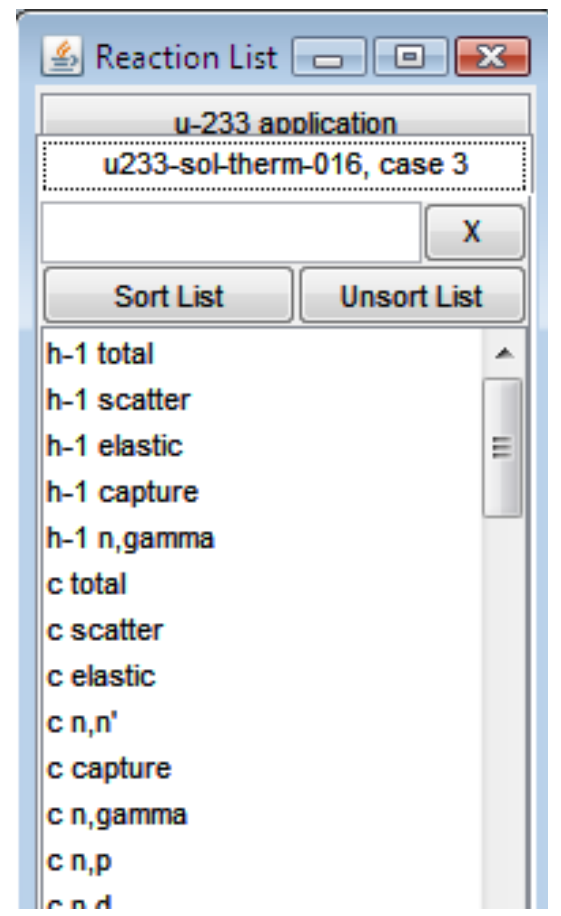

Fig. 35. Javapeño reaction list with data for ${ }^{233} \mathrm{U}$ application and U233-SOL-THERM-016-003.

First, plot the ${ }^{233} \mathrm{U}$ nubar sensitivity data for the application by selecting the application tab, scrolling to or filtering for u-233 nubar, right-clicking on u-233 nubar, and selecting Plot in new window from the pop-up menu, as shown in Fig. 36. Next, select the tab for the benchmark, filter for $\mathrm{u}-233$ nubar, and double-click u-233 nubar in the list, as shown in Fig. 37, to add the benchmark sensitivity data to the plot of the application sensitivity data. 


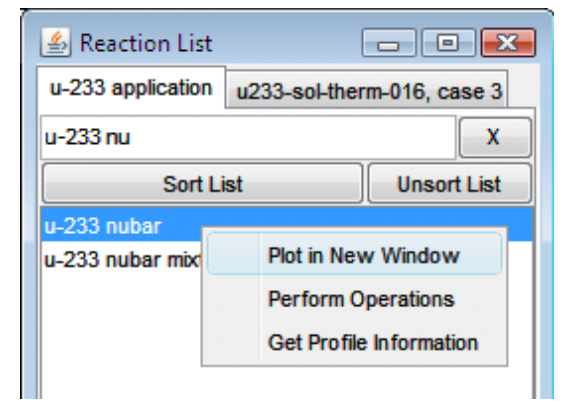

Fig. 36. Javapeño reaction list for plotting ${ }^{233} \mathrm{U}$ nubar sensitivity data for ${ }^{233} \mathrm{U}$ application in a new window.

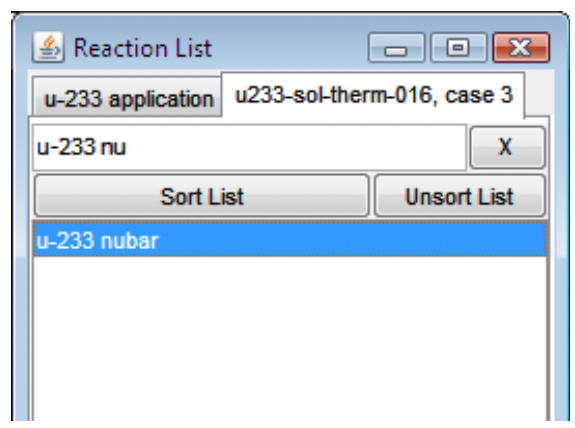

Fig. 37. Javapeño reaction list for plotting ${ }^{233} U$ nubar sensitivity data for U233-SOL-THERM-016-003.

The resulting plot with the two sensitivity profiles is shown in Fig. 38. The two profiles are nearly identical across the entire energy spectrum, indicating that these two systems should have very similar bias in term of ${ }^{233} \mathrm{U}$ nubar. Other nuclides and reactions for this or other systems can be inspected in a similar manner. 


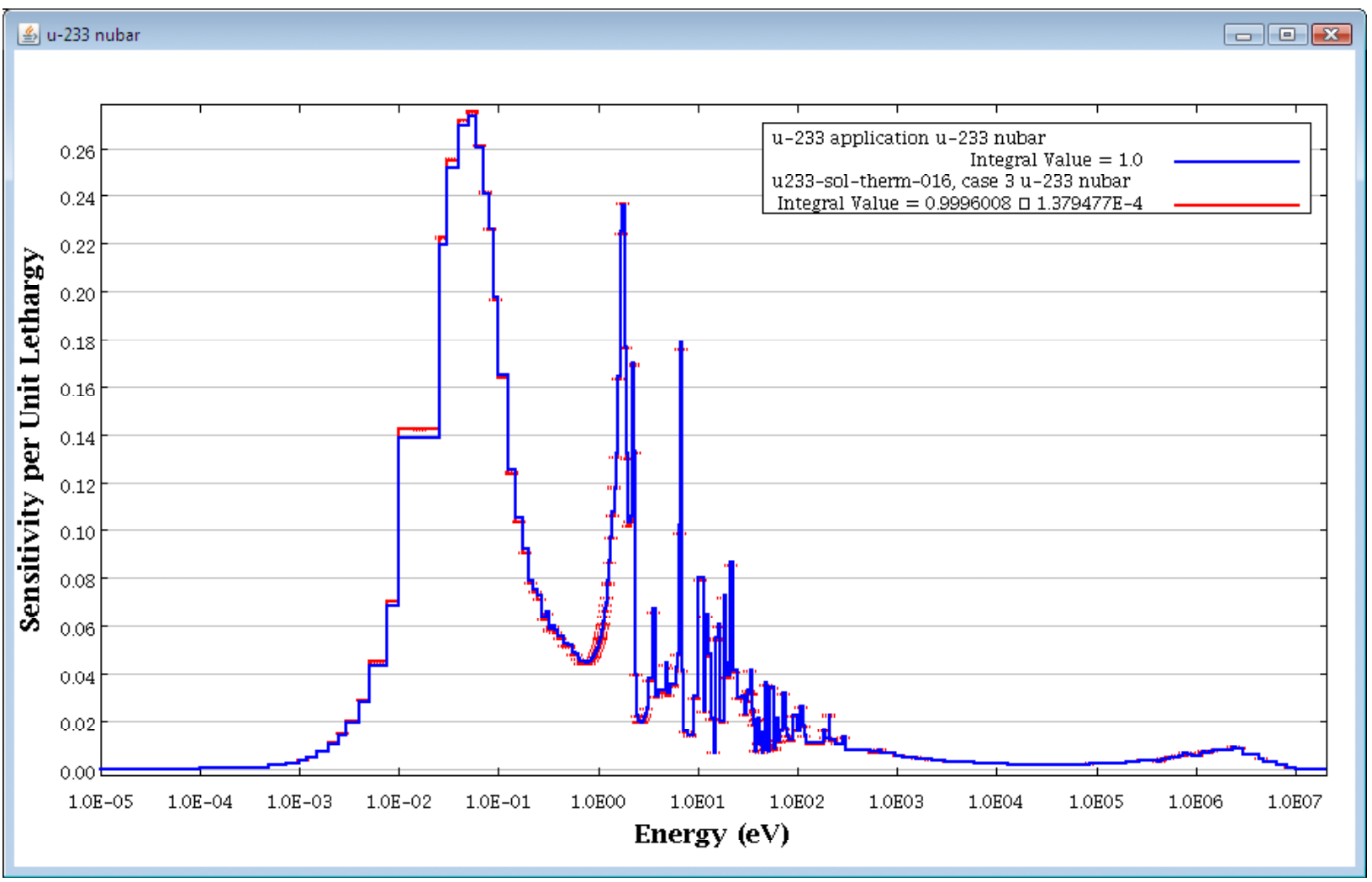

Fig. 38. Javapeño plot of ${ }^{233} \mathrm{U}$ nubar sensitivities for ${ }^{233} \mathrm{U}$ application and U233-SOL-THERM-016 case 3.

VIBE can also retrieve information from DICE to quickly provide descriptions of benchmarks identified with sensitivity data. To determine details of the all experiments from U233-SOL-THERM-016, remove the -003 from the filter on the handbook benchmark set, as shown in Fig. 39. Note that there are 31 benchmarks in this evaluation. Checking the benchmarks from the table that have ${ }^{233} \mathrm{U}$ nubar thermal sensitivities near 0.69 , only 11 of these 31 benchmarks from U233-SOL-THERM-016 meet the criteria.

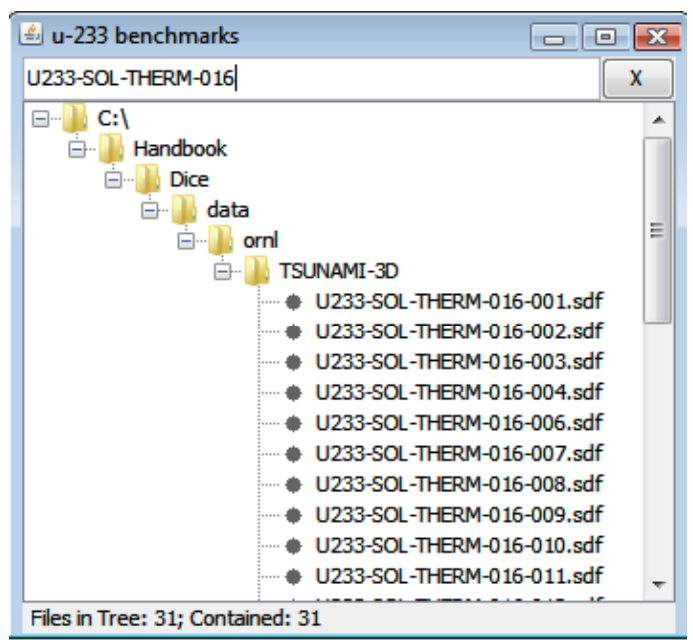

Fig. 39. Handbook benchmark set filtered for "U233-SOL-THERM-016." 
To find details from the DICE database for all 31 benchmarks in U233-SOL-THERM-016, select Retrieve data from DICE Database from the VIBE Action menu, as shown in Fig. 40. In the DICE query dialog, select Fuel from the left column, then check the boxes next to Fuel Concentration and Fuel Composition, as shown in Fig. 41. Under Benchmark Keff and Uncertainty, select Benchmark Keff and Benchmark Keff Uncertainty, as shown in Fig. 42, and then click View Data.

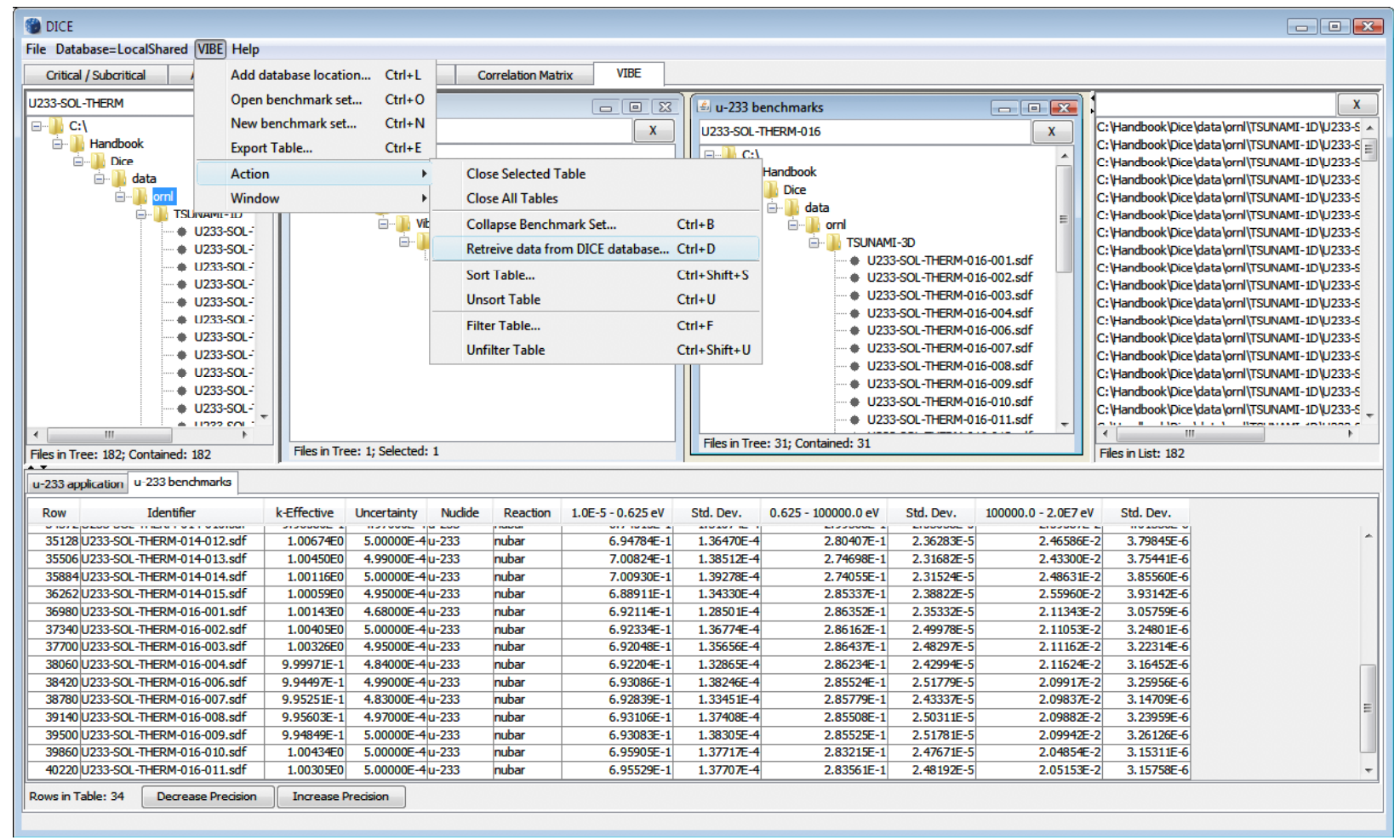

Fig. 40. Retrieve data from DICE Database menu option. 


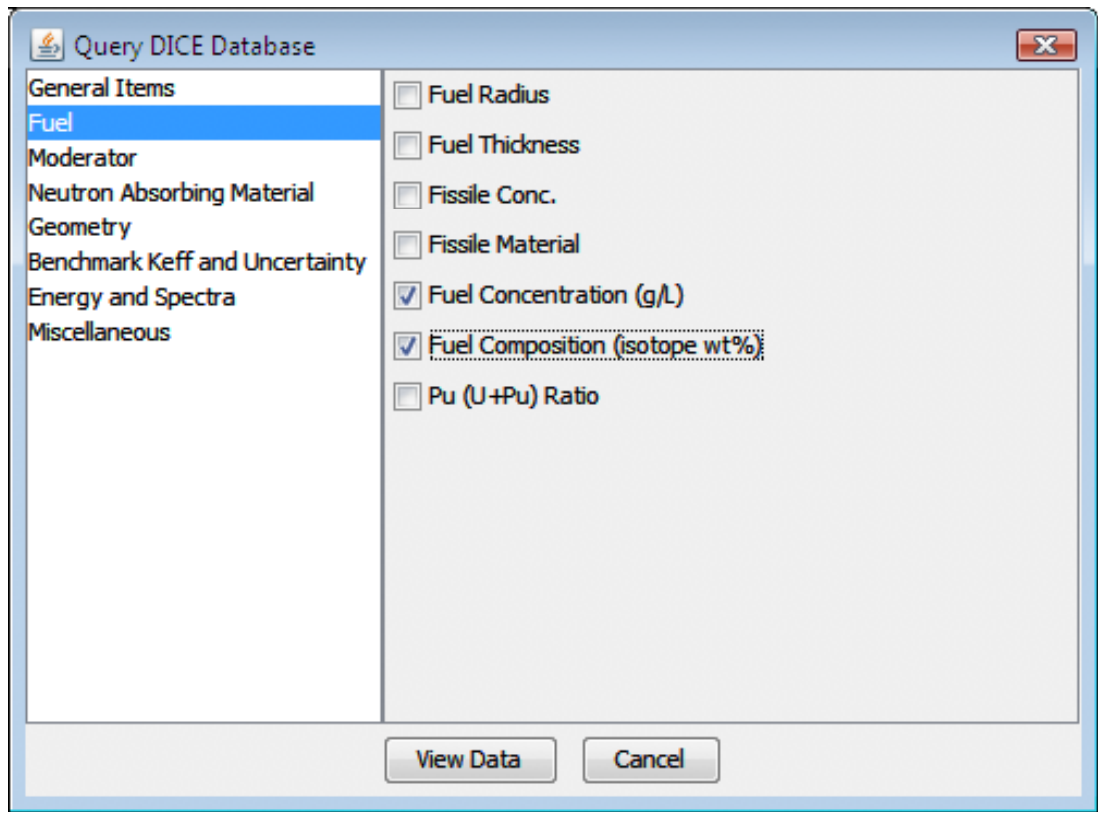

Fig. 41. Fuel options in DICE database query dialog.

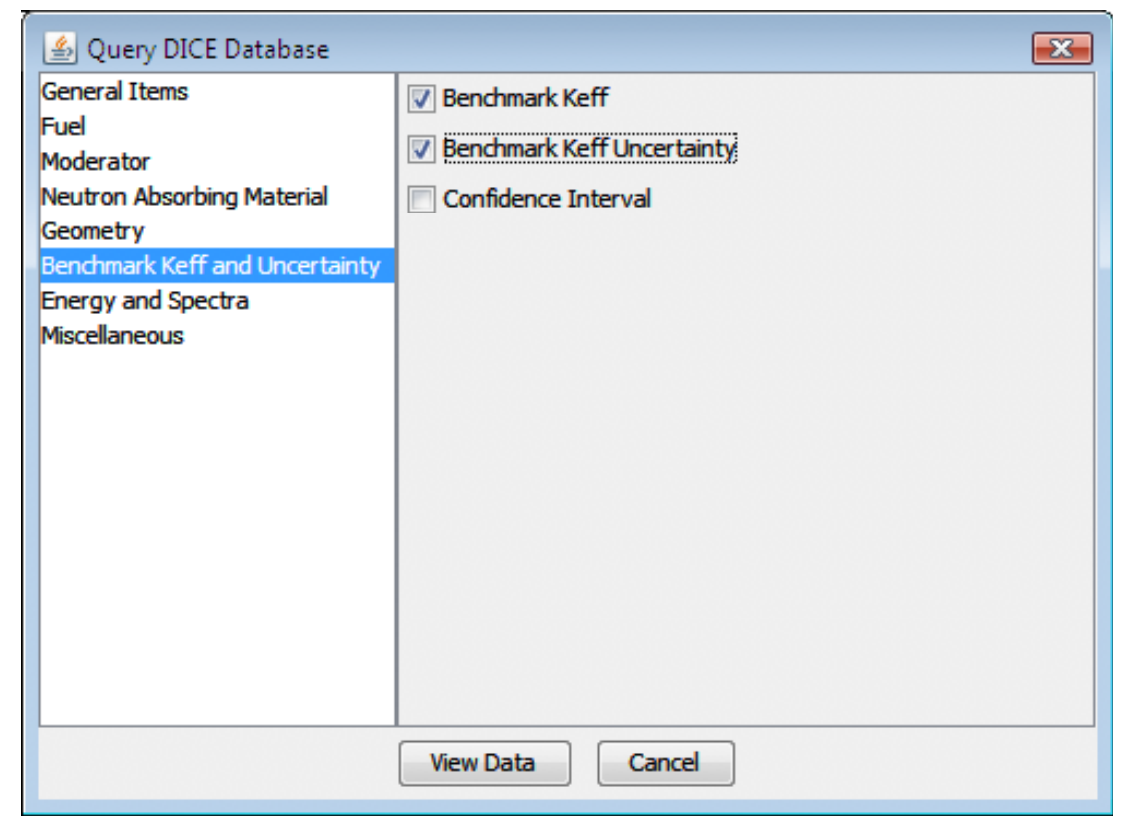

Fig. 42. Benchmark $\boldsymbol{k}_{\text {eff }}$ and uncertainty options in DICE database query dialog. 
A new table containing the results of the DICE query will be added to VIBE, as shown in Fig. 43. Here it can be observed that these experiments have a uranium content that is $97.5 \mathrm{wt} \%{ }^{233} \mathrm{U}$. Also, the solution concentration varies from 44.69 to $203.98 \mathrm{~g} / \mathrm{L}$. Recall that the application system was $100 \mathrm{wt} \%{ }^{233} \mathrm{U}$ with a concentration of $200 \mathrm{~g} / \mathrm{L}$. Note that the 11 cases identified as having the ${ }^{233} \mathrm{U}$ nubar thermal sensitivities near that of the application are the cases with solution concentrations of $200 \mathrm{~g} / \mathrm{L}$. The selection of the same 11 experiments based on TSUNAMI sensitivity criteria or solution concentration criteria confirms the validity of the TSUNAMI approach in comparison to more traditional approaches.

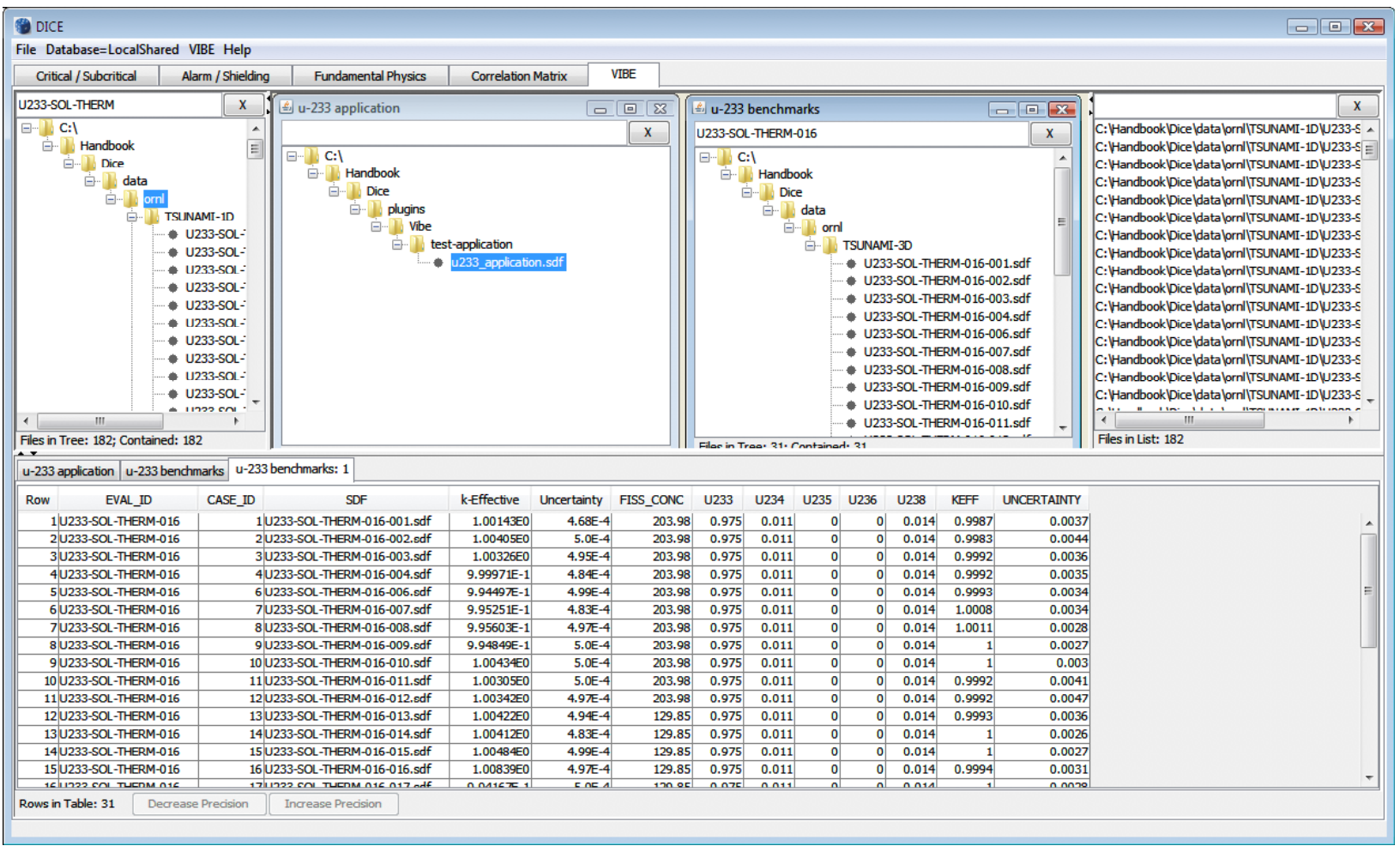

Fig. 43. VIBE with table of DICE query results.

U233-SOL-THERM experiments with solution concentrations between 180 and $220 \mathrm{~g} / \mathrm{L}$ can be identified with another VIBE query. Removing the benchmark identifier - 016 from the u-233 benchmarks window filter, as shown in Fig. 44, and retrieving the same data from the DICE database as shown in Fig. 41 and Fig. 42, and then filtering for FISS_CONC of 180-220, as shown in Fig. 45, reveals 29 matching cases, as shown in Fig. 46. Here U233-SOL-THERM-002 case 1 is shown to have a solution concentration of $200.45 \mathrm{~g} / \mathrm{L}$. However, TSUNAMI data showed matching ${ }^{233} \mathrm{U}$ nubar sensitivity for U233-SOL-THERM002 case 12 , not case 1 . Using another DICE query to examine all cases from U233-SOL-THERM-002, shown in Fig. 47 reveals that case 12 has a concentration of $277.585 \mathrm{~g} / \mathrm{L}$. 


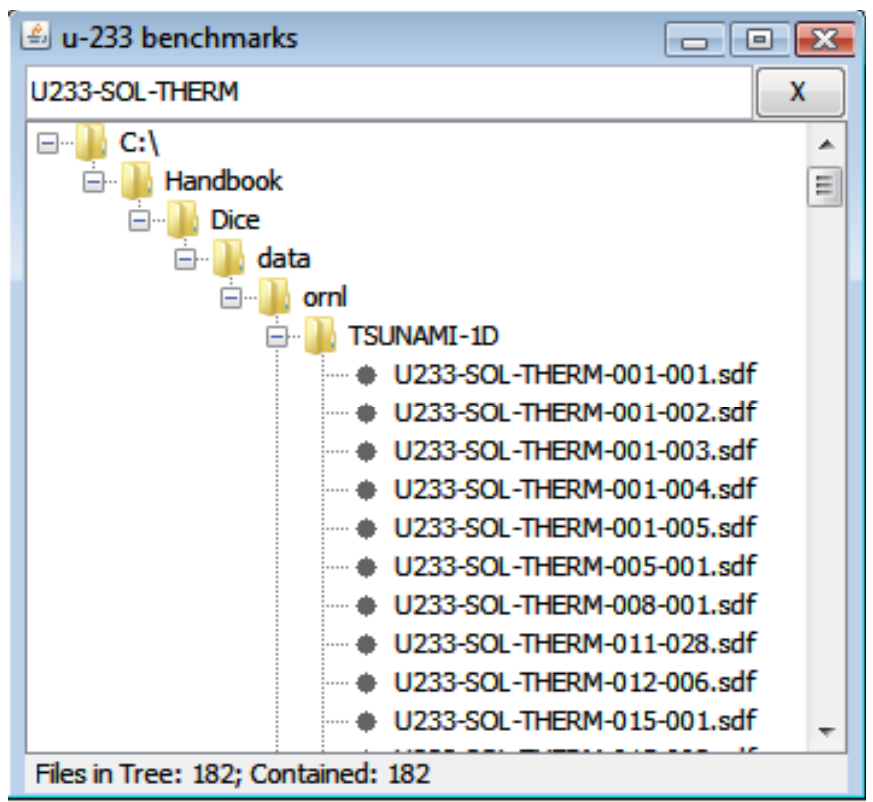

Fig. 44. Benchmarks window with U233-SOL-THERM filter applied.

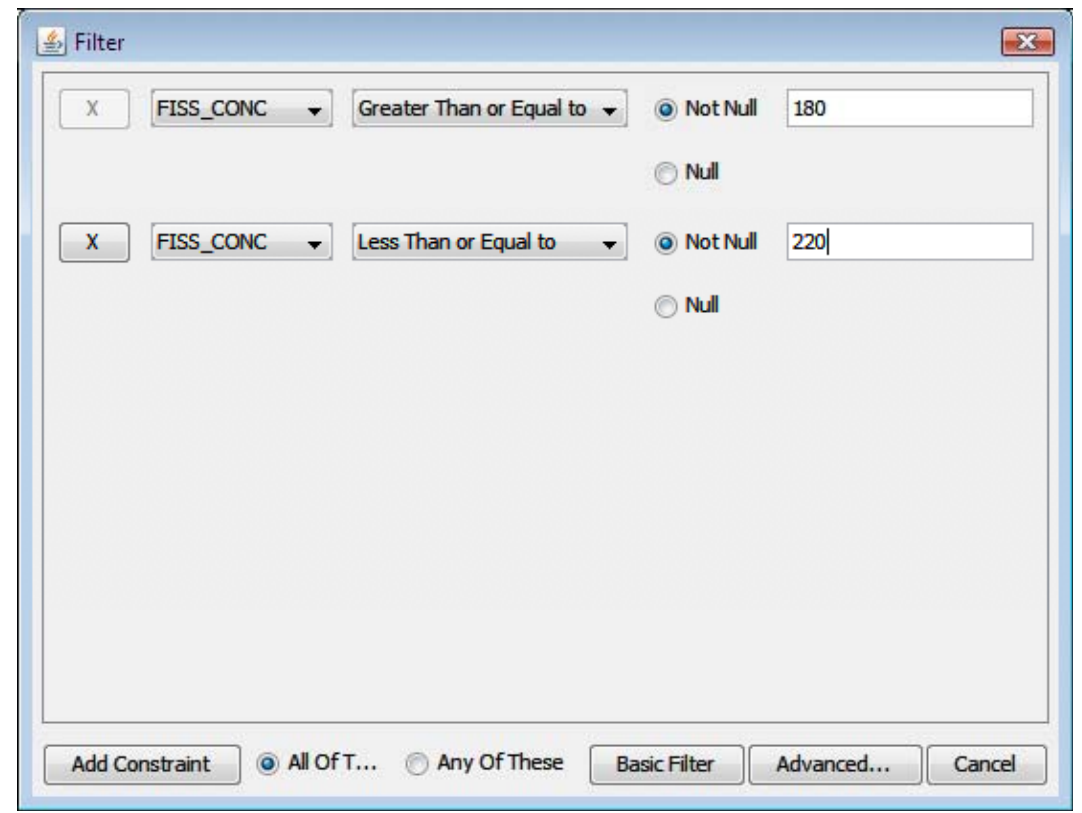

Fig. 45. Filter for fissile concentrations of $180-220 \mathrm{~g} / \mathrm{L}$. 


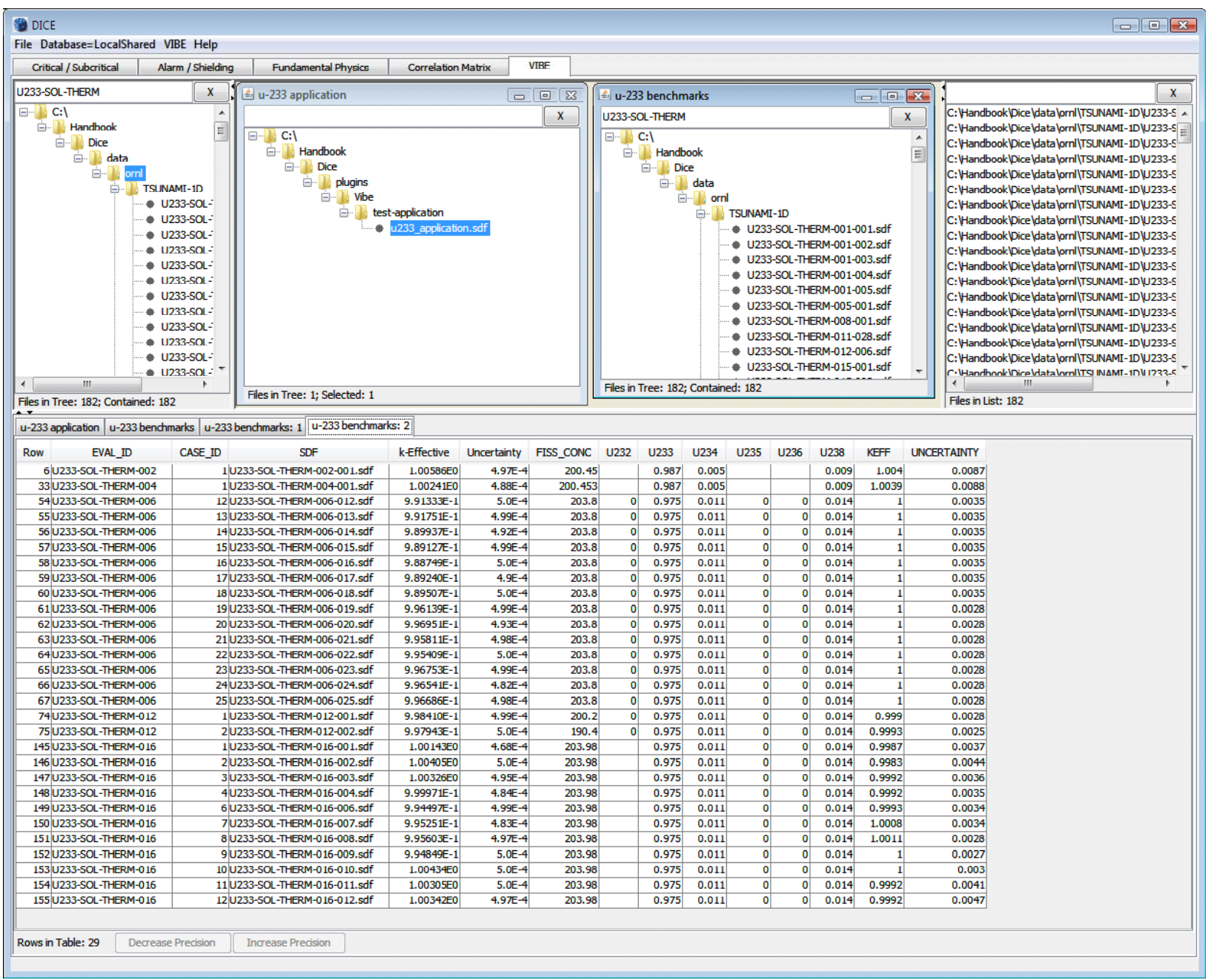

Fig. 46. VIBE DICE query results for all U233-SOL-THERM experiments filtered from concentrations near $200 \mathrm{~g} / \mathrm{L}$. 


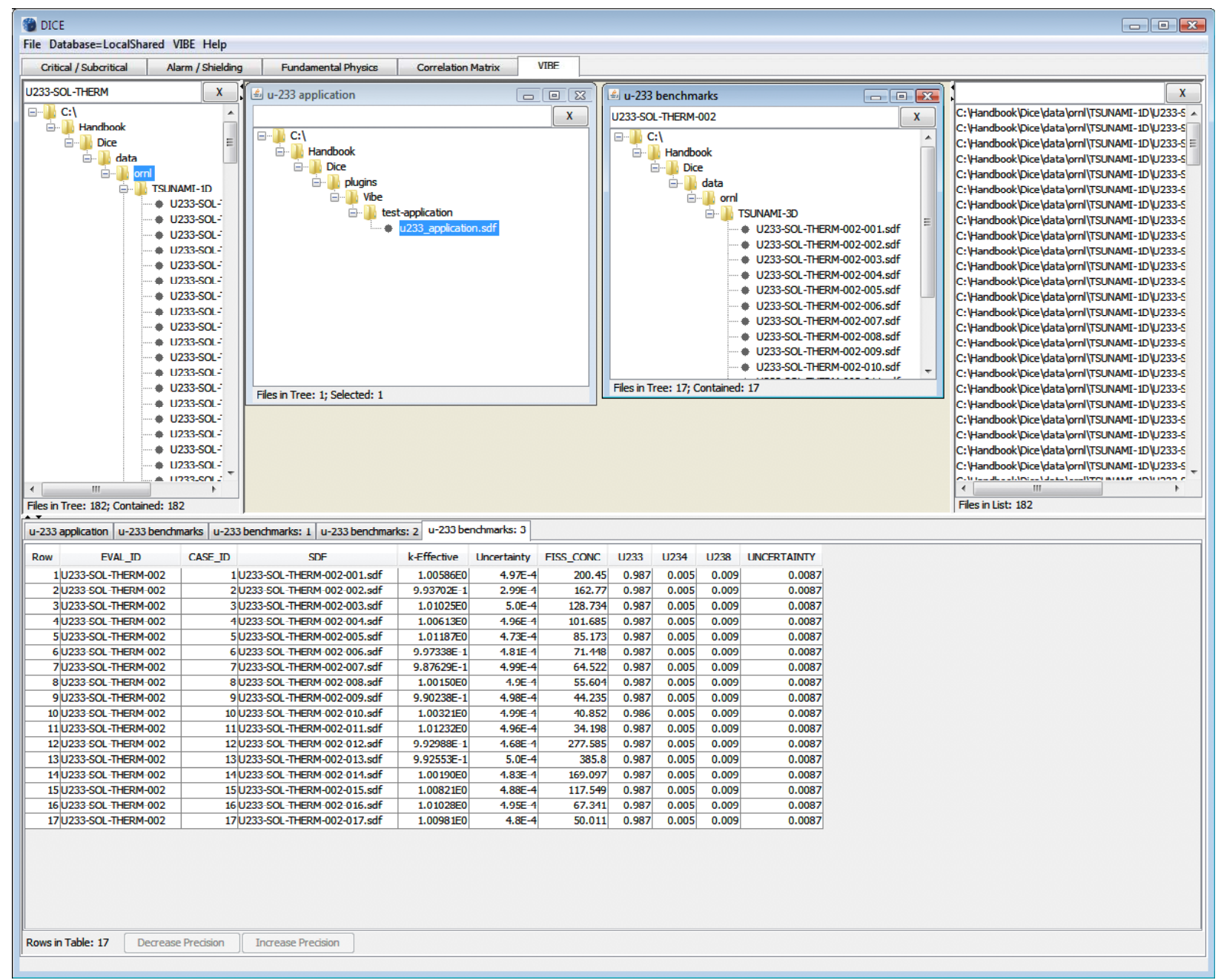

Fig. 47. VIBE DICE query results for U233-SOL-THERM-002 benchmarks.

Using VIBE to open the SDFs in Javapeño and then plotting ${ }^{233} \mathrm{U}$ nubar for the two benchmarks and the application reveal a higher sensitivity to ${ }^{233} \mathrm{U}$ nubar for case 1 than for case 12 , which more closely matches the sensitivity of the application, as shown in Fig. 48. In this case the moderation of the solutions is different enough to shift the spectra, causing the case with the higher solution concentration to more closely match the application. In this instance, a thorough and potentially time-consuming review of data from the handbook evaluation or the DICE database could have revealed these subtleties to an expert, where the TSUNAMI sensitivity data quickly revealed similarity in terms of the physics of the systems, not just the characteristics of the compositions. 


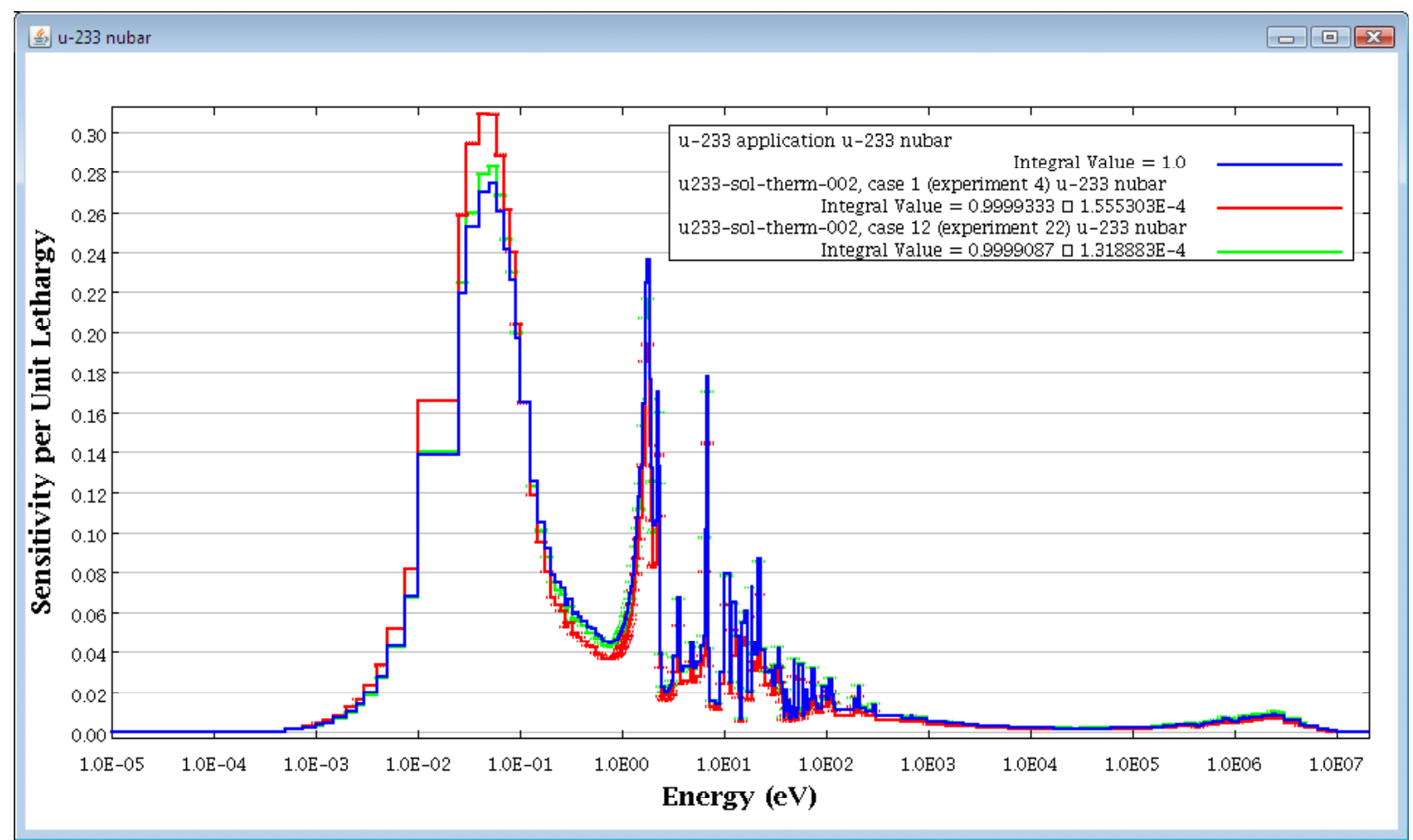

Fig. 48. Sensitivity profiles for ${ }^{233} \mathrm{U}$ nubar from ${ }^{233} \mathrm{U}$ application and U233-SOL-THERM-002 cases 1 and 12.

A final step in the use of VIBE is to export the desired files in a VIBE Data File (VDF) format suitable for more advanced analysis with TSUNAMI-IP (TSUNAMI Indices and Parameters) and TSURFER

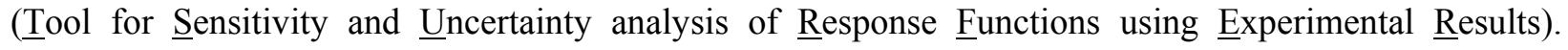
Remove any text from the filter field of the $\mathrm{u} 233$ benchmarks window in the center benchmark pane of VIBE so that all 182 U233-SOL-THERM benchmarks are listed. Right-click inside the u233 benchmarks window, and select Save Benchmark Set from the contextual menu, as shown in Fig. 49. Browse to a destination directory, ensure that the file type is set to VIBE Benchmark Sets ( . vdf), and click Save, as shown in Fig. 50. The VDF file options dialog will appear, as shown in Fig. 51. Checking the box next to Include benchmark k-eff and uncertainty data from DICE database will include the benchmark $k_{\text {eff }}$ and benchmark $k_{\text {eff }}$ uncertainty data required for bias assessment. A new file called u233 benchmarks. vdf will be saved to provide easy access to the benchmarks in the ExSITE interface of SCALE 6.

The interested reader is directed to the TSUNAMI Primer, ${ }^{5}$ available online at http://www.ornl.gov/sci/scale/tsunami/references.shtml for more information on the application of TSUNAMI to criticality code validation. 


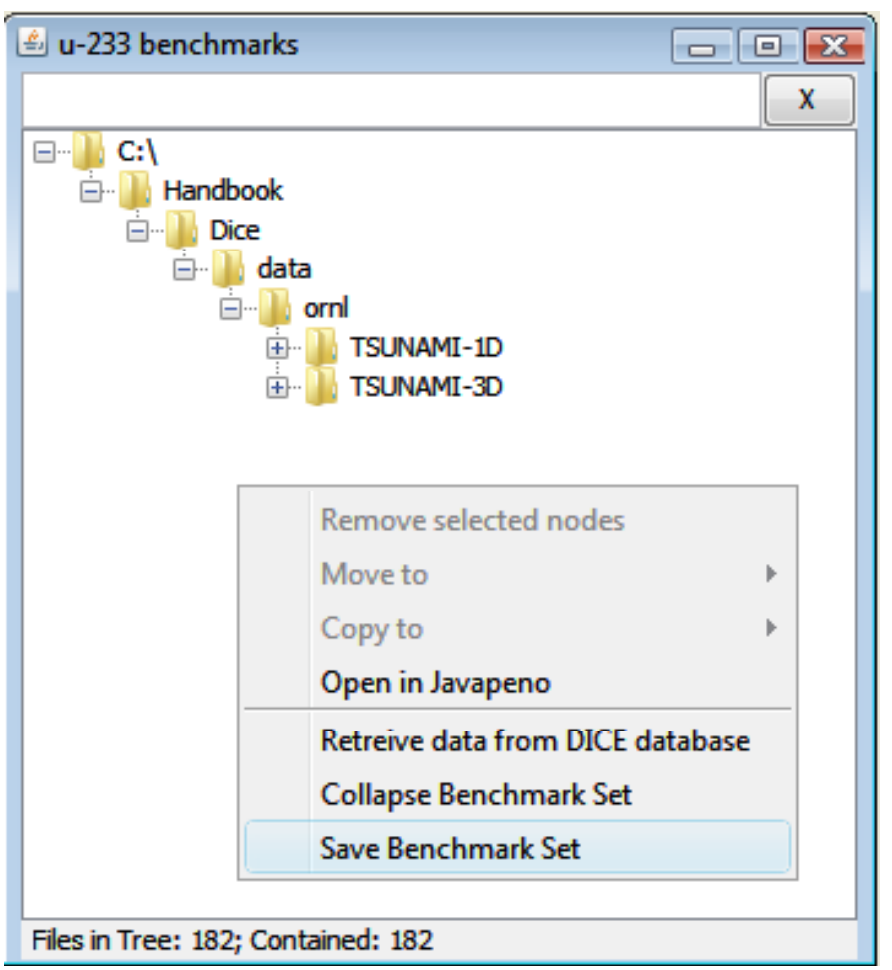

Fig. 49. Contextual menu to save benchmark sets.

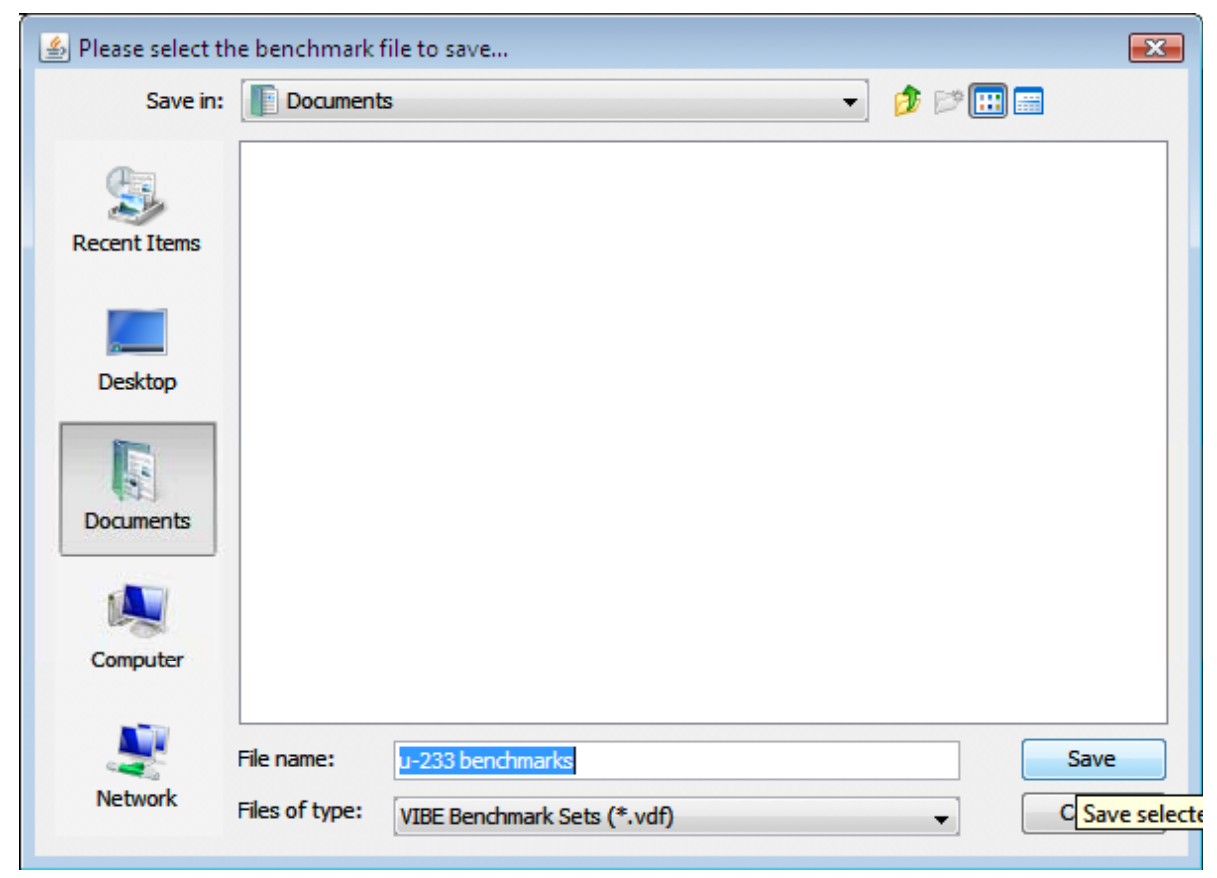

Fig. 50. Benchmark set save dialog. 


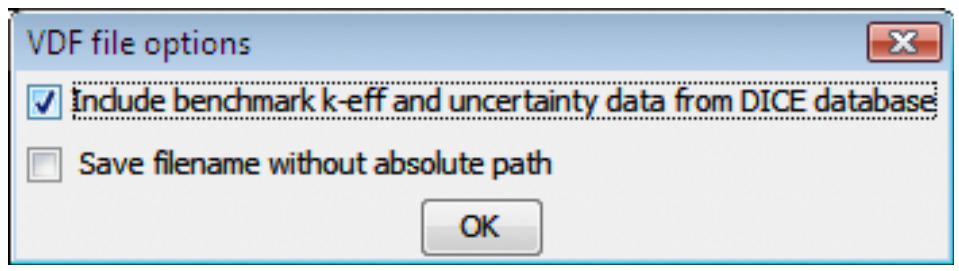

Fig. 51. VDF file options dialog. 


\section{EXPORTING GROUP-COLLAPSED SENSITIVITY DATA}

VIBE provides the user with the option of exporting group-collapsed sensitivity data from TSUNAMI to new sensitivity files. To perform a collapsed export, select a table of collapsed data and right-click on the title tab of the table and select Export..., as shown in Fig. 52. In the Export Table dialog, select As .sdf, as shown in Fig. 53. In the Exporting Collapsed SDFs dialog, shown in Fig. 54, the user can enter a text identifier, which will be added to the file name of each collapsed sensitivity file. Here the test_3group_ is used to identify the structure of the collapsed data. The location to store the collapsed files is also set in this dialog. Pressing Export will begin the process of exporting the files.

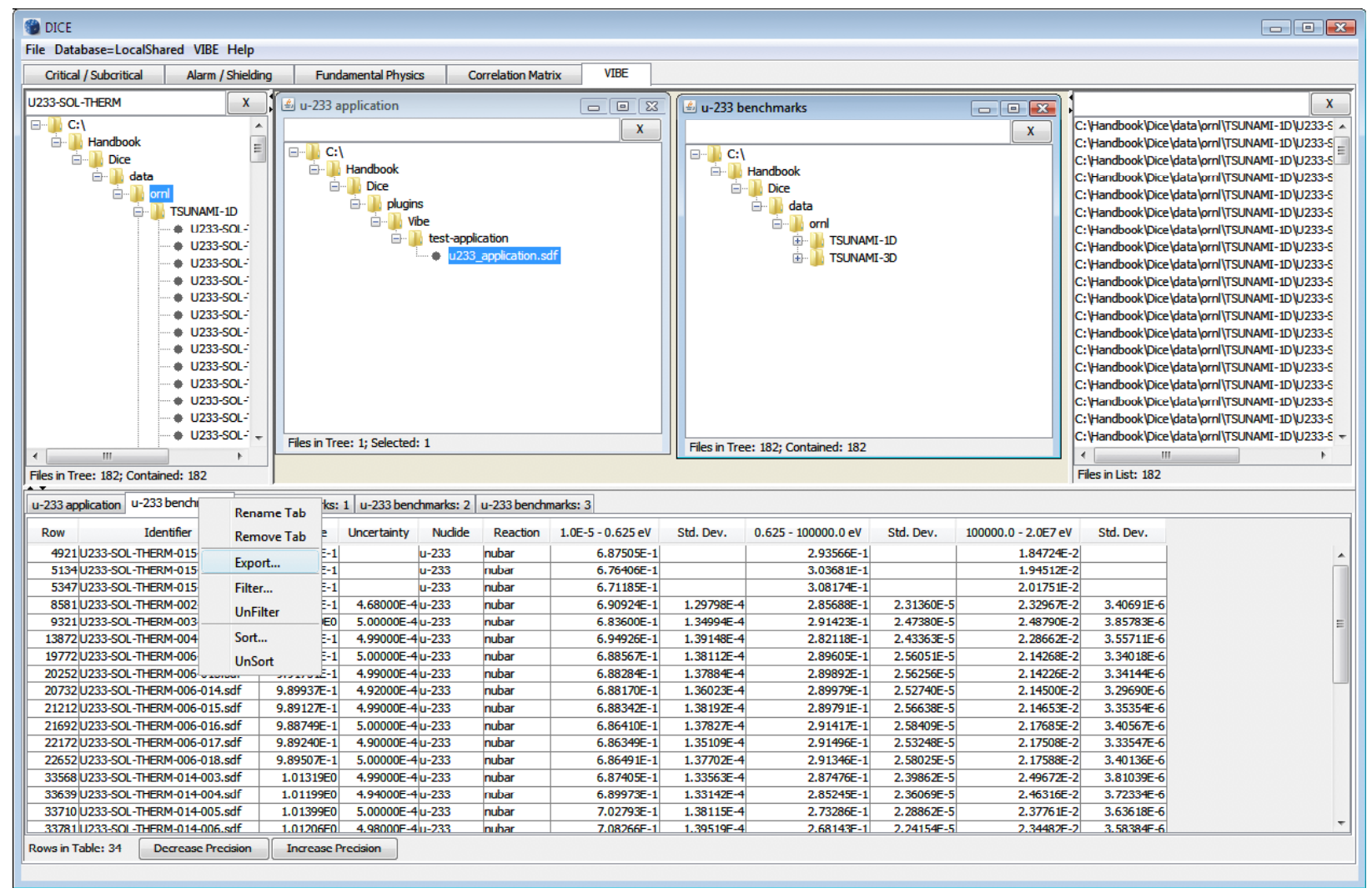

Fig. 52. Group-collapsed table contextual menu.

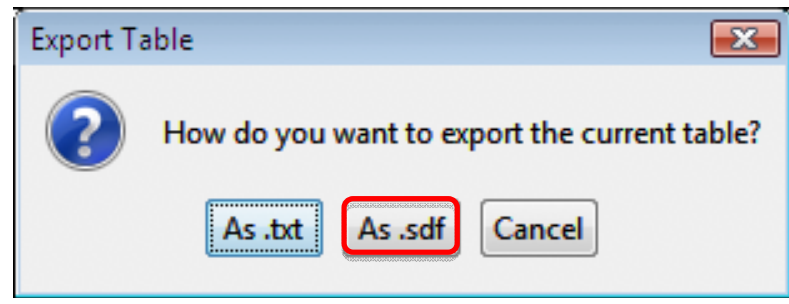

Fig. 53. Export table dialog. 


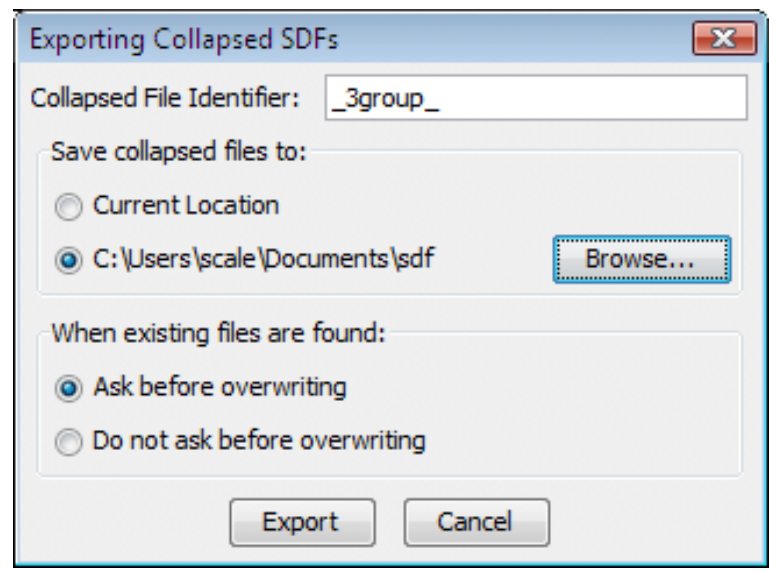

Fig. 54. Exporting collapsed SDFs dialog.

The collapsed sensitivity data are written to unique files corresponding to each file original sensitivity data file, as shown in Fig. 55. These data can be plotted in Javapeño and compared to the 238-group data, as shown in Fig. 56.

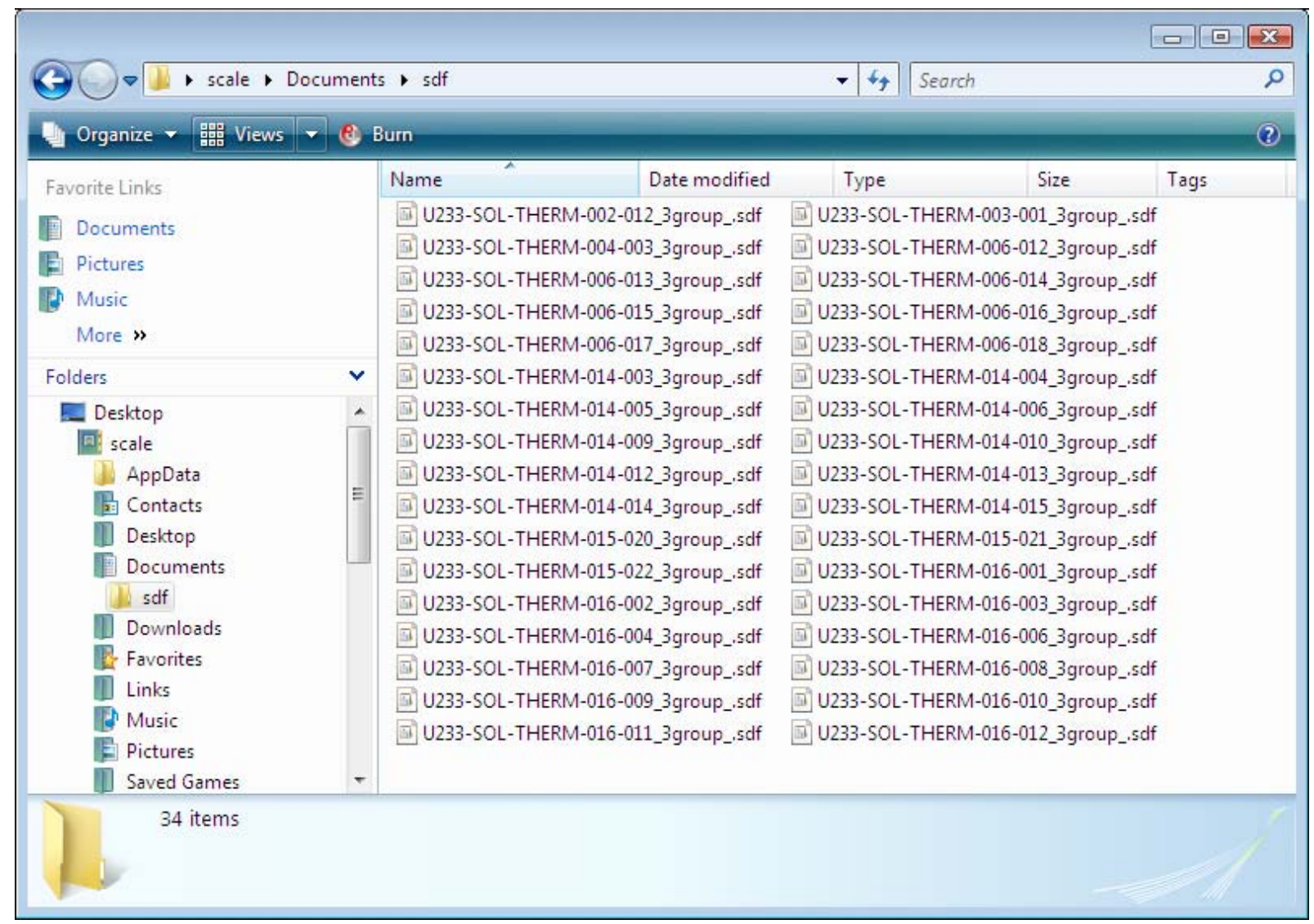

Fig. 55. Directory containing collapsed sensitivity file. 


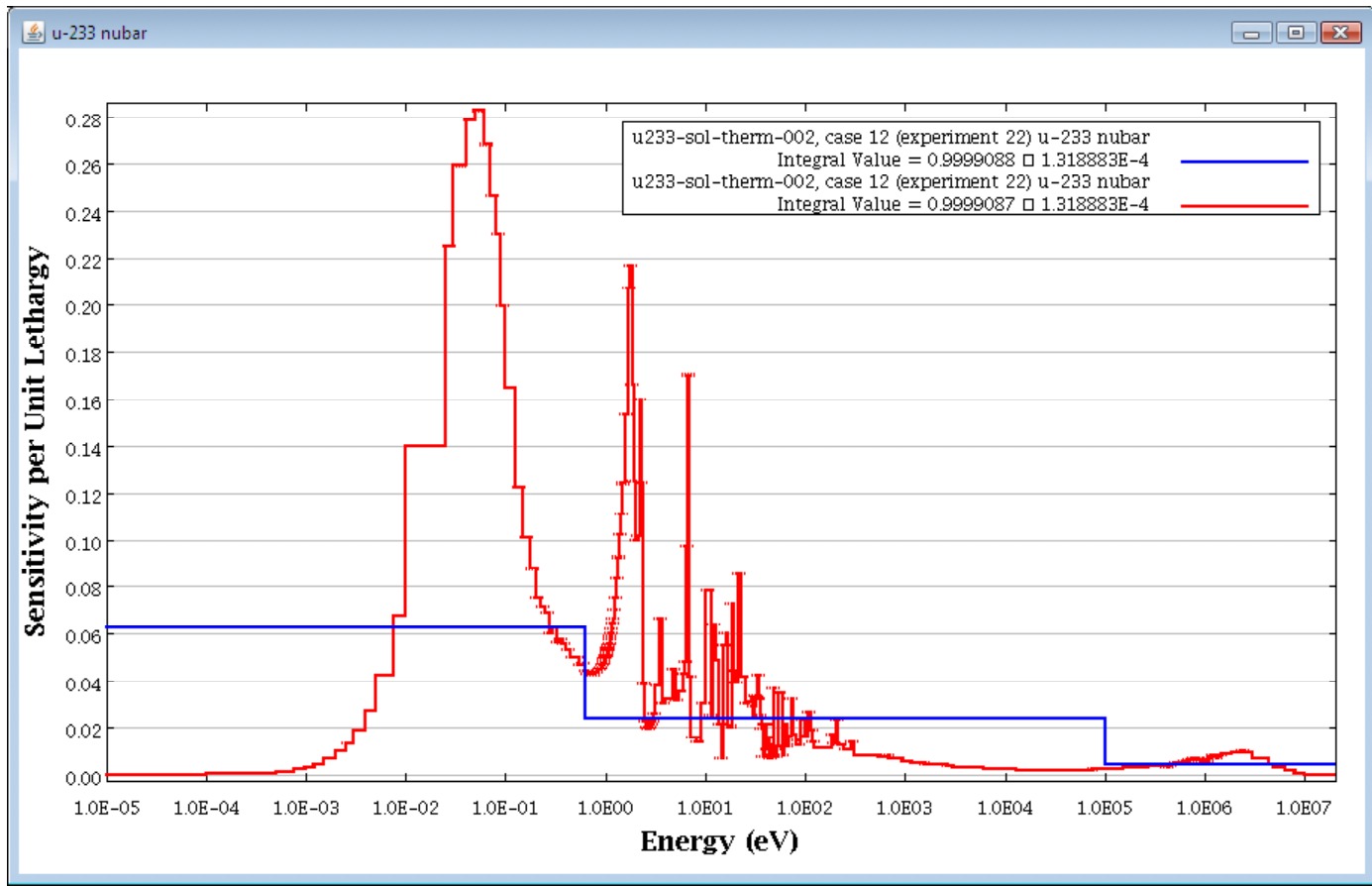

Fig. 56. Plot of 3- and 238-group sensitivity data. 



\section{CONCLUSIONS}

This guide has presented an example use of VIBE as a DICE plug-in to access and interpret the sensitivity data provided through the ICSBEP. The use of VIBE to identify important processes in an example application and identify benchmark experiments with similar sensitivities has been demonstrated. Information regarding the physical characteristics of these systems was retrieved from the DICE database.

Planned improvements for VIBE include quantification of similarity using sensitivity- and uncertaintybased integral indices, such as the TSUNAMI-IP $c_{k}$ parameter to quickly identify relevant benchmark experiments. Additionally, sufficient information is available in VIBE to perform parametric trending analyses to provide a first-cut computational bias and bias uncertainty assessment based on the computed $k_{\text {eff }}$ values included in the TSUNAMI sensitivity data files. Additional techniques for gap analysis to identify important processes in the application where no relevant benchmarks exist may also be incorporated.

Questions regarding the capabilities or uses of VIBE should be directed to ORNL by contacting scalehelp@ornl.gov. 



\section{REFERENCES}

1. B. T. Rearden, R. A. Lefebvre, A. B. Thompson, Y. Rugama, N. Soppera, and M. Bossant, "The VIBE Tool of SCALE - Validation, Interpretation and Bias Estimation," Proc. 2009 Nuclear Criticality Safety Division Topical Meeting on Realism, Robustness and the Nuclear Renaissance, NCSD 2009, Richland, WA, September 13-17, 2009.

2. SCALE: A Modular Code System for Performing Standardized Computer Analyses for Licensing Evaluation, ORNL/TM-2005/39, Version 6, Vols. I-III, Oak Ridge National Laboratory, Oak Ridge, Tennessee, January 2009. Available from Radiation Safety Information Computational Center at Oak Ridge National Laboratory as CCC-750.

3. International Handbook of Evaluated Criticality Safety Benchmark Experiments, NEA/NSC/DOC(95)03/I-VIII, OECD-NEA, September 2009.

4. D. E. Mueller, B. T. Rearden, and D. F. Hollenbach, Application of the SCALE TSUNAMI Tools for the Validation of Criticality Safety Calculations Involving ${ }^{233}$ U, ORNL/TM-2008/196, Oak Ridge National Laboratory, Oak Ridge, Tenn., January 2009.

5. B. T. Rearden, D. E. Mueller, S. M. Bowman, R. D. Busch, and S. J. Emerson, TSUNAMI Primer: A Primer for Sensitivity/Uncertainty Calculations with SCALE, ORNL/TM-2009/027, Oak Ridge National Laboratory, Oak Ridge, Tenn., January 2009. 